\title{
The Power Spectrum of Galaxy Clustering in the Las Campanas Redshift Survey
}

\section{Citation}

Lin, Huan, Robert P. Kirshner, Stephen A. Shectman, Stephen D. Landy, Augustus Oemler, Douglas L. Tucker, and Paul L. Schechter. 1996. "The Power Spectrum of Galaxy Clustering in the Las Campanas Redshift Survey." The Astrophysical Journal 471 (2): 617-35. https:// doi.org/10.1086/177993.

\section{Permanent link}

http://nrs.harvard.edu/urn-3:HUL.InstRepos:41399925

\section{Terms of Use}

This article was downloaded from Harvard University's DASH repository, and is made available under the terms and conditions applicable to Other Posted Material, as set forth at http:// nrs.harvard.edu/urn-3:HUL.InstRepos:dash.current.terms-of-use\#LAA

\section{Share Your Story}

The Harvard community has made this article openly available.

Please share how this access benefits you. Submit a story.

Accessibility 
ThE AstrophysiCAL JournaL, 471:617-635, 1996 November 10

(C) 1996. The American Astronomical Society. All rights reserved. Printed in U.S.A.

\title{
THE POWER SPECTRUM OF GALAXY CLUSTERING IN THE LAS CAMPANAS REDSHIFT SURVEY
}

\author{
HuAN LIN ${ }^{1}$ AND ROBERT P. KIRSHNER \\ Harvard-Smithsonian Center for Astrophysics, 60 Garden Street, Cambridge, MA 02138; kirshner@cfa.harvard.edu \\ STEPHEN A. SHeCTMAN AND STEPHEN D. LANDY \\ Carnegie Observatories, 813 Santa Barbara Street, Pasadena, CA 91101; shec@ociw.edu, landy@ociw.edu \\ Augustus OemLer \\ Department of Astronomy, Yale University, New Haven, CT 06520-8101; oemler@astro.yale.edu \\ DOUGLAS L. TUCKER \\ Astrophysikalisches Institut Potsdam, An der Sternwarte 16, D-14482 Potsdam, Germany; dtucker@aip.de \\ AND \\ PAUl L. SChEChTER \\ Department of Physics, Massachusetts Institute of Technology, Cambridge, MA 02139; schech@achernar.mit.edu \\ Received 1996 February 12; accepted 1996 June 10
}

\begin{abstract}
The Las Campanas Redshift Survey (LCRS) contains 23,697 galaxies, with an average redshift $z=0.1$, distributed over six $1.5 \times 80^{\circ}$ slices in the north and south galactic caps. We have computed the power spectrum $P(k)$ for magnitude-limited samples of LCRS galaxies over wavelengths $\lambda=2 \pi / k=5-400 h^{-1}$ Mpc. The LCRS $P(k)$ may be approximated as $\propto k^{-1.8 \pm 0.1}$ for small scales $\lambda=5-30 h^{-1} \mathrm{Mpc}$, changing to $\propto k^{1 \pm 1}$ for large scales $\lambda \approx 200-400 h^{-1} \mathrm{Mpc}$. The overall amplitude corresponds to $\sigma_{8}=1.0 \pm 0.1$ in redshift space.

Comparisons to the power spectra of other redshift surveys will be presented; the LCRS results agree best with those from the combined Center for Astrophysics (CfA2) and Southern Sky redshift surveys (SSRS2). For $\lambda \gtrsim 100 h^{-1} \mathrm{Mpc}$, the LCRS results are consistent with those of other surveys, given the large errors among all the surveys on these scales. For $\lambda \lesssim 100 h^{-1}$ Mpc, the LCRS $P(k)$ is well determined and similar in shape to the $P(k)$ of other surveys, but with an amplitude differing from some of the other samples, possibly because of inherent clustering differences among different types of galaxies. In particular, power spectrum measurements for volume-limited LCRS samples show that galaxies brighter than about $M^{*}-1$ appear about $50 \%$ more strongly clustered than those fainter. Also, a sample of LCRS emission galaxies shows $30 \%$ weaker clustering than the full LCRS sample.

Comparisons to $N$-body models show that the LCRS power spectrum lies intermediate between that of a standard flat $\Omega_{0} h=0.5$ cold dark matter (CDM) model and an open $\Omega_{0} h=0.2$ model, both normalized to $\sigma_{8}=1$ for galaxies. On large scales $\lambda \gtrsim 40 h^{-1} \mathrm{Mpc}$, we have fit the LCRS results to various linear CDM models, and find that a number of them could meet the constraints set by the LCRS power spectrum, the Hubble constant range $0.5 \lesssim h \lesssim 0.8$, the abundance of galaxy clusters, and the reasonable assumption that LCRS galaxies are roughly unbiased tracers of the mass, relative to the normalization provided by the 4 year COBE DMR data. The possibilities include open CDM or flat nonzero cosmological-constant CDM models with $\Omega_{0} \approx 0.4-0.6$ and shape parameter $\Gamma \approx \Omega_{0} h \approx 0.2-0.3$, as well as flat $\Omega_{0}=1$ models with massive neutrino density $\Omega_{v} \approx 0.2-0.3$ or a spectral tilt $n \approx 0.7-0.8$.

Subject headings: cosmology: observations - galaxies: clusters: general galaxies: distances and redshifts - large-scale structure of universe methods: numerical
\end{abstract}

\section{INTRODUCTION}

The power spectrum of density fluctuations is an important fundamental quantity of interest for the problem of structure formation in cosmology. For example, for Gaussian density fluctuations, as may arise out of inflationary scenarios for the origin of the universe (Bardeen, Steinhardt, $\&$ Turner 1983), the power spectrum provides a complete description of the initial fluctuations. The subsequent development and evolution of structure via gravitational instability, in the context of a dark matter dominated universe and models of biased galaxy formation (Bardeen et al.

\footnotetext{
${ }^{1}$ Present Affiliation: Department of Astronomy, University of Toronto, 60 St. George Street, Toronto, ON M5S 3H8, Canada; lin@astro.utoronto.ca.
}

1986), have received much theoretical attention in the past decade, both analytically and through the use of large numerical $N$-body simulations (e.g., Davis et al. 1985; Park 1991; Zurek et al. 1994). On the observational front, the detection of microwave background anisotropies by the COBE DMR experiment (Smoot et al. 1992) provided important constraints on the primordial power spectrum on horizon scales. The clustering of galaxies on smaller scales and the interpretation of the observations to reveal the matter content of the universe, to elucidate the process of structure formation, and to connect to the primordial fluctuations seen by $C O B E$, remain important problems in observational cosmology (see, e.g., Lyth \& Liddle 1996 for a review).

Much recent attention has been paid toward determination of the power spectrum of galaxy clustering in a 
variety of galaxy surveys, including the CfA (Vogeley et al. 1992; Park et al. 1994), SSRS (Park, Gott, \& da Costa 1992; da Costa et al. 1994b), IRAS 1.2 Jy (Fisher et al. 1993), IRAS QDOT (Feldman, Kaiser, \& Peacock 1994), and APM (Baugh \& Efstathiou 1993, 1994) surveys. In brief, the power spectra of these surveys have appeared inconsistent with predictions of the "standard" biased cold dark matter (CDM) model of structure formation with $\Omega_{0} h=0.5$ (Blumenthal et al. 1984), while an unbiased $\Omega_{0} h \approx 0.2$ model with more large-scale power agrees better with the observations (e.g., da Costa et al. 1994b). (We express the Hubble constant as $H_{0}=100 h \mathrm{~km} \mathrm{~s}^{-1} \mathrm{Mpc}^{-1}$, and will use $h=1$ unless otherwise indicated.) In this paper we present the power spectrum for galaxy samples drawn from the Las Campanas Redshift Survey (LCRS), an optically selected survey of 23,697 galaxies with an average redshift $z=0.1$. The large sample size and extent of our survey allow us to examine the power spectrum up to wavelengths of $\approx 400 h^{-1} \mathrm{Mpc}$, and to provide measurements independent of previous results for the purpose of comparing against cosmological models. In particular, measurements of the power spectrum on the largest scales $\lambda \gtrsim 100 h^{-1}$ Mpc are especially interesting, as we expect the power spectrum to peak there and begin its turnover toward the primordial spectrum constrained by $C O B E$ and other microwave background observations. The precise amplitude and shape of the power spectrum on large scales will provide important clues in discriminating among cosmological models.

A detailed description of the Las Campanas survey is given in Shectman et al. (1996), and additional particulars may be found in Shectman et al. $(1992,1995)$, Tucker (1994), Lin et al. (1996), and Oemler et al. (1993). Here we briefly describe the main survey parameters. The survey geometry is that of six $1.5 \times 80^{\circ}$ "slices" (declination by right ascension), three each in the north and south galactic caps. Figure 1 shows the LCRS galaxy distribution and clearly illustrates the striking pattern of clusters, filaments, walls and voids that is present. The first $20 \%$ of the data was obtained using a 50 object fiber-optic spectrograph, and the remaining $80 \%$ of the data was taken with a 112 object system. The nominal isophotal magnitude limits for the 50 fiber data were $16.0 \leq m<17.3$ ("hybrid" Kron-Cousins $R$ magnitudes), and an additional cut was applied that excluded the lowest $20 \%$ of galaxies by central surface brightness. For the 112 fiber data, the nominal magnitude limits were $15.0 \leq m<17.7$, with exclusion of just the lowest $4 \%-9 \%$ of galaxies by surface brightness. The survey photometric limits were chosen so that there would be typically more targets per field than available fibers, and we selected targets at random among those that met the selection criteria. The survey slices were built up by observing $1.5 \times 1.5$ fields, one at a time, with a maximum of 50 or 112 galaxies observed per field. Because we generally do not reobserve any of our fields, we must keep track of the variable field-to-field sampling fractions $f$ in our subsequent statistical analyses. The average sampling fraction is $70 \%$ for the 112 fiber data and $58 \%$ for the 50 fiber data. Also, mechanical constraints prevent two object fibers in a single spectroscopic field from approaching closer than $55^{\prime \prime}$, introducing an additional geometric selection effect. We will find below that the various sampling, photometric, and geometric selection effects in our survey do not significantly affect the power spectrum results.

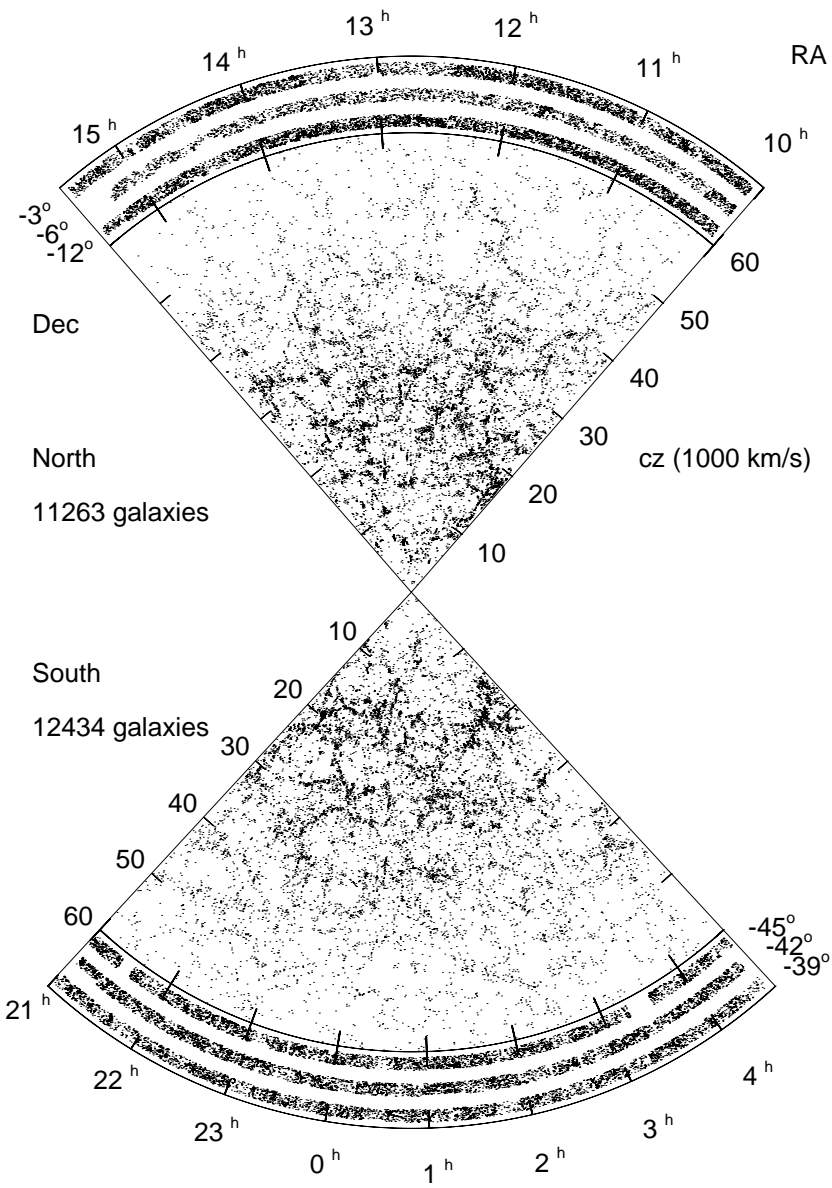

FIG. 1.-LCRS galaxy distribution in the northern and southern galactic caps.

In $\S 2$ we detail our power spectrum estimation techniques and verify them on $N$-body simulations. In $\S 3$ we present the power spectra of magnitude-limited samples of Las Campanas galaxies, and compare our results to the power spectra derived from other redshift surveys. In $\S 4$ we compute the power spectrum for volume-limited samples of Las Campanas galaxies and test for luminosity bias in the survey. In $\S 5$ we compare our power spectrum results against those from $N$-body simulations. We will then focus on the large-scale linear power spectrum, relate our results to the $C O B E$ DMR constraints, and compare against the predictions of several classes of CDM models. We summarize our results in $\S 6$. Note that a complementary analysis of the two-dimensional LCRS power spectrum has already been carried out (Landy et al. 1996; more on this below), and the derivation of the closely related two-point correlation function of LCRS galaxies is described in Tucker (1994) and Tucker et al. (1996).

\section{ESTIMATING THE POWER SPECTRUM}

The power spectrum estimation technique used here has been described by various authors - in particular see Fisher et al. (1993), Feldman et al. (1994), and Park et al. (1994) - and we detail the method below. The most important difference is that the LCRS consists of six essentially two-dimensional "slices," so that we need to account for "convolution" effects caused by the survey geometry in order to calculate the power spectrum properly. These convolution effects are also evaluated below. 


\subsection{Methods}

Given a galaxy density field $\rho(\boldsymbol{r})$ with density contrast $\delta(\boldsymbol{r})=[\rho(\boldsymbol{r})-\bar{\rho}] / \bar{\rho}$, where $\bar{\rho}$ is the mean galaxy density, we have the two-point correlation function

$$
\xi(\boldsymbol{r})=\left\langle\delta\left(\boldsymbol{r}^{\prime}\right) \delta\left(\boldsymbol{r}^{\prime}+\boldsymbol{r}\right)\right\rangle_{\boldsymbol{r}^{\prime}}
$$

and the power spectrum

$$
P(\boldsymbol{k})=\int d^{3} \boldsymbol{r} \xi(\boldsymbol{r}) e^{i \boldsymbol{k} \cdot \boldsymbol{r}} .
$$

With this definition $P(\boldsymbol{k})$ is a power spectral density in units of volume. Assuming isotropy we have $\xi(\boldsymbol{r})=\xi(r=|\boldsymbol{r}|)$ and $P(\boldsymbol{k})=P(k=|k|)$.

Now suppose we have a survey of volume $V$, with $N$ observed galaxies at positions $\boldsymbol{r}_{i}$. The survey's selection function $s(\boldsymbol{r})$ is defined to be the fraction of galaxies at position $r$ expected to be observable by the survey, given the survey's photometric and other selection criteria. Also, let $w(\boldsymbol{r}) \equiv 1 / \mathrm{s}(\boldsymbol{r})$. We can then form an estimator $\hat{\delta}(\boldsymbol{r})$ for the true density contrast $\delta(\boldsymbol{r})$ by

$$
\hat{\delta}(\boldsymbol{r})=\frac{1}{\sum_{i=1}^{N} w\left(\boldsymbol{r}_{i}\right) / V} \sum_{i=1}^{N} w\left(\boldsymbol{r}_{i}\right) \delta^{(3)}\left(\boldsymbol{r}-\boldsymbol{r}_{i}\right)-1,
$$

where $\delta^{(3)}$ is the Dirac delta function and where we have estimated the mean density $\bar{\rho}$ by $\sum_{i=1}^{N} w\left(\boldsymbol{r}_{i}\right) / V$. Note that we will use a circumflex ${ }^{\wedge}$ to denote quantities estimated from the observations. We next take the Fourier transform

$$
\begin{aligned}
\hat{\delta}(\boldsymbol{k}) & \equiv \frac{1}{V} \int d^{3} \boldsymbol{r} \hat{\delta}(\boldsymbol{r}) e^{i \boldsymbol{k} \cdot \boldsymbol{r}} \\
& =\frac{1}{\sum_{i=1}^{N} w\left(\boldsymbol{r}_{i}\right)} \sum_{i=1}^{N} w\left(\boldsymbol{r}_{i}\right) e^{i \boldsymbol{k} \cdot \boldsymbol{r}_{i}}-W(\boldsymbol{k}),
\end{aligned}
$$

where $W(\boldsymbol{k})$ is the survey window function

$$
W(\boldsymbol{k}) \equiv \frac{1}{V} \int_{V} d^{3} \boldsymbol{r} e^{i \boldsymbol{k} \cdot \boldsymbol{r}} .
$$

We can convert the sum over galaxies in equation (5) to a sum over infinitesimal cells distributed throughout the survey volume, as in Peebles (1980, $\S 36$ and 41), where each cell $i$ holds $n_{i}=1$ or 0 galaxies. Then the expectation value of $|\hat{\delta}(\boldsymbol{k})|^{2}$ may be written

$$
\begin{aligned}
\left\langle|\hat{\delta}(\boldsymbol{k})|^{2}\right\rangle= & \frac{1}{(\bar{\rho} V)^{2}} \sum_{i} \sum_{j}\left\langle n_{i} n_{j}\right\rangle w\left(\boldsymbol{r}_{i}\right) w\left(\boldsymbol{r}_{j}\right) e^{i \boldsymbol{k} \cdot\left(\boldsymbol{r}_{i}-\boldsymbol{r}_{j}\right)} \\
& +|W(\boldsymbol{k})|^{2} \\
& -\frac{1}{\bar{\rho} V} \sum_{i}\left\langle n_{i}\right\rangle w\left(\boldsymbol{r}_{i}\right)\left[e^{i \boldsymbol{k} \cdot \boldsymbol{r}_{i}} W^{*}(\boldsymbol{k})+e^{-i \boldsymbol{k} \cdot \boldsymbol{r}_{i}} W(\boldsymbol{k})\right] .
\end{aligned}
$$

Using equations (2) and (6), and the relations

$$
\left\langle n_{i} n_{j}\right\rangle= \begin{cases}\bar{\rho}^{2}\left[1+\xi\left(\left|\boldsymbol{r}_{i}-\boldsymbol{r}_{j}\right|\right)\right] s\left(\boldsymbol{r}_{i}\right) s\left(\boldsymbol{r}_{j}\right) d V_{i} d V_{j} & i \neq j, \\ \bar{\rho} s\left(\boldsymbol{r}_{i}\right) d V_{i} & i=j,\end{cases}
$$

we can rewrite equation (7) as

$$
\left\langle|\hat{\delta}(\boldsymbol{k})|^{2}\right\rangle=\frac{\tilde{P}(\boldsymbol{k})}{V}+\langle S\rangle,
$$

which is the sum of the true power spectrum convolved with the survey window function,

$$
\tilde{P}(\boldsymbol{k}) \equiv \frac{V}{(2 \pi)^{3}} \int d^{3} \boldsymbol{k}^{\prime} P\left(k^{\prime}\right)\left|W\left(\boldsymbol{k}-\boldsymbol{k}^{\prime}\right)\right|^{2},
$$

and the expectation value of the shot noise arising from sampling a finite sample of galaxies,

$$
\langle S\rangle \equiv \frac{1}{\bar{\rho} V^{2}} \int d^{3} r w(r) .
$$

Note that we are using a tilde $(\sim)$ to denote the convolved power spectrum. For a given set of galaxies the shot noise $S$ is given exactly by the $i=j$ terms in the first sum of equation (7),

$$
S=\frac{1}{\left[\sum_{i=1}^{N} w\left(\boldsymbol{r}_{i}\right)\right]^{2}} \sum_{i=1}^{N} w^{2}\left(\boldsymbol{r}_{i}\right),
$$

and our first estimate of the observed power spectrum, convolved with the survey window function, is thus

$$
\hat{\tilde{P}}(k)=V \int \frac{d \Omega_{k}}{4 \pi}\left[|\hat{\delta}(k)|^{2}-S\right],
$$

where we average over different wavevectors $k$ at fixed magnitude $|\boldsymbol{k}|=k$. However, because we do not know the true mean density beforehand, but must estimate it from the survey itself, we will underestimate the power spectrum on scales comparable to the survey size by an amount $\widetilde{P}(0)|W(k)|^{2}$ (Peacock \& Nicholson 1991). It turns out that for our survey geometry, $\widetilde{P}(k) \approx$ constant $\approx \widetilde{P}(0)$ on large scales (see the next subsection). Given an initial estimate for $\widetilde{P}(0)$, we can correct for the underestimate by adding back the $\tilde{P}(0)|W(k)|^{2}$ term, so that our final estimate for the observed convolved power spectrum is

$$
\hat{\tilde{P}}(k)=\int \frac{d \Omega_{\boldsymbol{k}}}{4 \pi}\left\{V\left[|\hat{\delta}(\boldsymbol{k})|^{2}-S\right]+\tilde{P}(0)|W(\boldsymbol{k})|^{2}\right\} .
$$

We limit our power spectrum computations to scales for which this correction is $\lesssim 10 \%$, which corresponds to $\lambda \lesssim$ $400 h^{-1} \mathrm{Mpc}$ for the LCRS. Now the expectation value of $\hat{\tilde{P}}(k)$ is, using equation $(10)$,

$$
\begin{aligned}
\langle\hat{\tilde{P}}(k)\rangle & =\int \frac{d \Omega_{k}}{4 \pi} \tilde{P}(k) \\
& =\int d k^{\prime} k^{\prime 2} P\left(k^{\prime}\right) K\left(k, k^{\prime}\right),
\end{aligned}
$$

where

$$
K\left(k, k^{\prime}\right) \equiv \frac{V}{(2 \pi)^{3}} \int \frac{d \Omega_{k}}{4 \pi} \int d \Omega_{\boldsymbol{k}^{\prime}}\left|W\left(\boldsymbol{k}-\boldsymbol{k}^{\prime}\right)\right|^{2} .
$$

The effect of the convolution integral (16) for our survey will be illustrated in the next subsection. Because convolution effects are large for our survey, it will be convenient to deconvolve the observed $\hat{\widetilde{P}}(k)$ to recover $P(k)$. We will use the iterative method due to Lucy (1974), which has been applied in a similar context by Baugh \& Efstathiou (1993) to recover the spatial power spectrum from the angular correlation function. Given the observed convolved power $\widehat{\widetilde{P}}$ at a set of wavenumbers $k_{j}$, we can find the true power $P$ at the wavenumbers $k_{i}$ by starting with initial guesses $P^{0}\left(k_{i}\right)$, 
and then computing new estimates $P^{n}\left(k_{i}\right)$ by iteration

$$
P^{n+1}\left(k_{i}\right)=P^{n}\left(k_{i}\right) \frac{\sum_{j}\left[\hat{\tilde{P}}\left(k_{j}\right) / \tilde{P}^{n}\left(k_{j}\right)\right] K\left(k_{i}, k_{j}\right) \Delta k}{\sum_{j} K\left(k_{i}, k_{j}\right) \Delta k},
$$

where

$$
\tilde{P}^{n}\left(k_{j}\right)=\sum_{l} k_{l}^{2} P^{n}\left(k_{l}\right) K\left(k_{j}, k_{l}\right) \Delta k .
$$

We also apply a smoothing procedure

$$
P^{n}\left(k_{i}\right) \rightarrow 0.5 P^{n}\left(k_{i}\right)+0.25\left[P^{n}\left(k_{i-1}\right)+P^{n}\left(k_{i+1}\right)\right],
$$

in order to reduce noise as the $P^{n}\left(k_{i}\right)$ converge to a final solution. On the largest scales $k \lesssim 0.03 h \mathrm{Mpc}^{-1}$, the smoothing also reduces the sensitivity of the solution to the choice of initial guess $P^{0}\left(k_{i}\right)$ (we adopt $P^{0}\left(k_{i}\right)=$ constant). Because the deconvolution procedure is not too well constrained on large scales, and because the smoothing procedure is somewhat arbitrary, we will use the deconvolved power spectra only for qualitative comparisons. The computation of the convolved power spectrum from a given true power spectrum is more straightforward, and our quantitative analyses will focus on the convolved power spectrum.

\subsection{Test of the Methods on $N$-Body Simulations}

We check our power spectrum estimation methods on an $N$-body simulation kindly provided by Changbom Park. The simulation is an open cold dark matter model (denoted ODM), with $\Omega_{0}=0.4$ and $h=0.5$, whose power spectrum has been found to be a good match to that of the Center for Astrophysics (CfA) Redshift Survey (Park et al. 1994). The model is unbiased, that is, $b=1$, where $b$ is the ratio of galaxy to mass density fluctuations. The model is normalized so that $\sigma_{8}=1$, where $\sigma_{8}$ is the rms galaxy density fluctuation in a sphere of radius $8 h^{-1} \mathrm{Mpc}$. The ODM simulation contains $240^{3}$ particles and was computed using a particle-mesh code (Hockney \& Eastwood 1981) on a $480^{3}$ mesh with corresponding physical comoving volume $\left(576 h^{-1} \mathrm{Mpc}\right)^{3}$.

We draw from the ODM simulation a set of "mock" redshift surveys using the geometric and photometric selection criteria of our actual survey (see $\S 3.1$ ). The ODM "galaxies" are assigned absolute magnitudes using the luminosity function derived for the LCRS (Lin et al. 1996). The velocities of the ODM galaxies are included so we measure the power spectrum in redshift space. For each mock survey we rederive the selection function and then compute the power spectrum using the methods detailed in $\S 2.1$. Now even though the ODM simulation box is quite large, the full LCRS still only fits along a body diagonal of the ODM box. We can, however, fit either the northern or southern halves of the survey into the box fairly readily, and we have drawn a series of northern and southern LCRS mock surveys. To make a reasonably large number of whole-LCRS mock surveys, we chose to combine a northern and a southern mock survey even though these two mock surveys are not originally oriented relative to each other in the simulation box as the real northern and southern surveys are. Figure 2 shows the average power spectrum measured from 30 whole-LCRS mock surveys. We show both the directly measured, or convolved, power, as well as the deconvolved power of the mock surveys. The errors plotted are the standard deviations of the mean (sdom) of

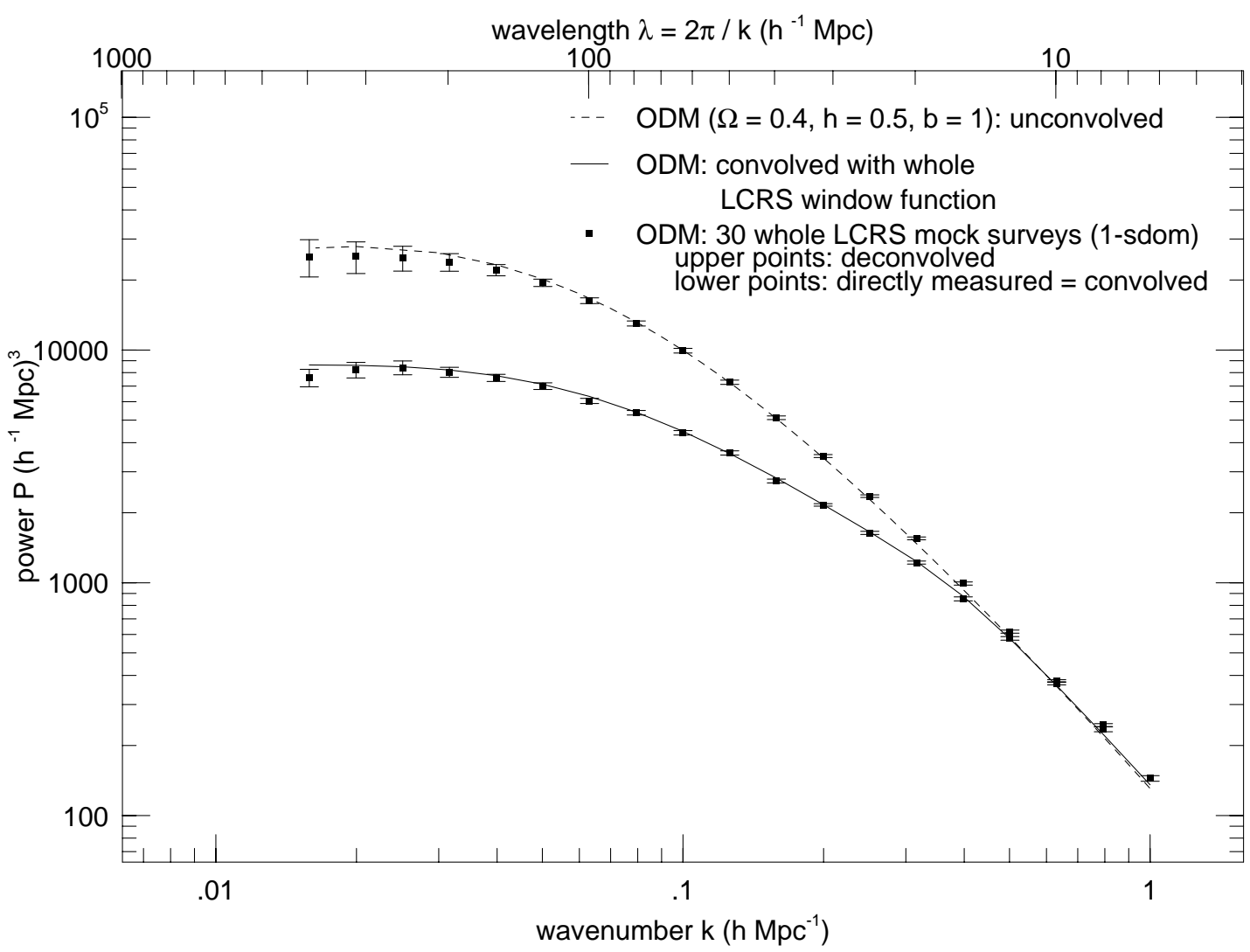

FIG. 2.- True unconvolved and convolved power spectra of the ODM $N$-body model and the corresponding power spectra as measured from the ODM LCRS mock surveys. The error bars are one standard deviation of the mean (sdom) as measured from the mock surveys. 
the 30 surveys. Also plotted in the figure are the true unconvolved power spectrum of the ODM simulation and the ODM power spectrum after convolution with our wholesurvey window function (using eqs. [16] and [17]). There is good agreement between the true ODM power spectrum and the average power spectrum of the mock surveys over the range of scales $\lambda=5-400 h^{-1} \mathrm{Mpc}$ that we examine, showing that we can correctly account for the geometry and selection criteria of the LCRS in our measurement and deconvolution of the mock-survey power spectra.

Note from Figure 2 that the main effect of the convolution by the window function of our survey is that the convolved power spectrum lies below the unconvolved power spectrum over most of the scales probed. Also, note that on the largest scales, wavelengths $\lambda>100 h^{-1} \mathrm{Mpc}$, the convolved power spectrum is flat, whereas the true power spectrum may peak and begin to turn over. (This is seen more clearly for the power spectrum of Fig. 6 below.) In Figure 3 we illustrate the convolution effect in more detail by plotting the integrand $k^{\prime 2} P\left(k^{\prime}\right) K\left(k, k^{\prime}\right)$ of the convolution integral (16) for the ODM model at several values of $k$. This integrand shows the contribution to the convolved power $\widetilde{P}$ at $k$ from the true power $P$ at different $k^{\prime}$. For a threedimensional survey geometry, such as a sphere or cube, at scales $\lambda=2 \pi / k$ small compared to the survey size, we would expect this integrand to be sharply peaked at $k^{\prime}=k$; that is, convolution effects would not be important and the measured convolved power would be very close to the true power. However, the Las Campanas survey has essentially a two-dimensional geometry: it is large in two dimensions but thin along the third (cf. Fig. 1). Convolution effects can be noticeably important at scales smaller than the survey size, and "aliasing" occurs: power at $k^{\prime} \neq k$ makes a significant contribution to the convolved power $\widetilde{P}$ at $k$, leading to the effects noticed from Figure 2. As seen in Figure 3, for $k \lesssim$ $0.1 \mathrm{~h} \mathrm{Mpc}^{-1}$, the integrand is broad and somewhat complicated in shape, including contributions from a large range of $k^{\prime}$. The situation improves for smaller scales and larger $k$, as the integrand becomes more and more sharply peaked at $k^{\prime}=k$. Nevertheless, though these convolution effects are complicated in detail, they can be computed and accounted for in the power spectrum analysis, as we saw earlier.

\section{POWER SPECTRUM OF LCRS GALAXIES}

\subsection{Power Spectrum of LCRS Samples}

We compute the power spectrum in redshift space, but first we convert our heliocentric redshifts to comoving distances $r$, assuming $\Omega_{0}=1$. Using a different value for $\Omega_{0}$ does not make much difference; for example, $r$ changes by $2 \%$ at $z=0.1$ if we instead use $\Omega_{0}=0.2$. We first consider the power spectrum for magnitude-limited samples of the LCRS. We thus need to weight by the inverse of the selection function, but we note that this weighting becomes large when the selection function becomes small at large distances, where only the intrinsically brightest and rarest galaxies are observed. Also, because of the surface brightness cuts imposed on the sample, we do not probe the galaxy luminosity function well at the faint end (Lin et al. 1996), but these intrinsically faint galaxies could make a large contribution in the weighting. To reduce the sensitivity of our power spectrum results to these potential problems, we

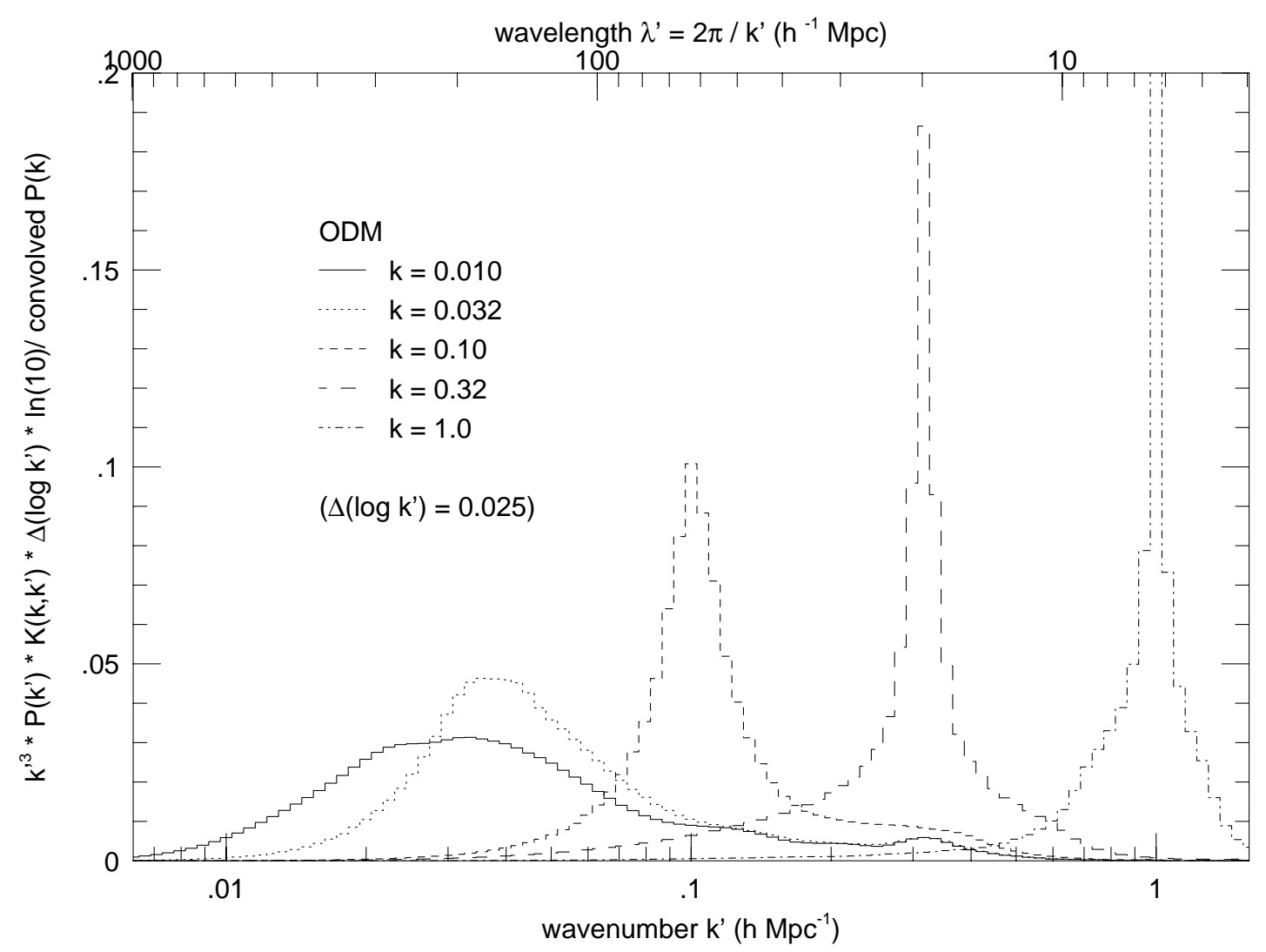

Fig. 3.-Integrand $k^{\prime 2} P\left(k^{\prime}\right) K\left(k, k^{\prime}\right)$ of the convolution integral (16) for the ODM model at five values of $k$. Note we have multiplied the integrand by $k^{\prime} \ln 10 \Delta\left(\log _{10} k^{\prime}\right)$ as appropriate for our log-linear plot, and we have also normalized by dividing by $\widetilde{P}(k)$. 
make additional restrictions on the redshifts and absolute magnitudes of the galaxies used in our clustering analyses: $10,000 \mathrm{~km} \mathrm{~s}^{-1}<c z<45,000 \mathrm{~km} \mathrm{~s}^{-1}$ (or $98<r<405 h^{-1}$ $\mathrm{Mpc})$ and $M_{1} \equiv-22.5<M-5 \log h<-18.5 \equiv M_{2}$. These limits keep the selection function $s(c z) \gtrsim 0.05$ and result in a total sample of 19,305 galaxies. The weight function $w$ used in equation (5) for galaxy $i$ of the survey is given by

$$
w\left(\boldsymbol{r}_{i}\right)=\frac{W_{i}}{s\left(\boldsymbol{r}_{i}\right)},
$$

where $W_{i}$ is a weighting factor that accounts for variable field-to-field sampling fractions and incompletenesses as a function of apparent magnitude and central surface brightness (details in Lin et al. 1996), and $s\left(\boldsymbol{r}_{i}\right)$ is the selection function appropriate to the survey field containing galaxy $i$,

$$
s\left(\boldsymbol{r}_{i}\right)=\int_{\max \left[M_{1}\left(\boldsymbol{r}_{i}\right), M_{1}\right]}^{\min \left[M_{2}\left(\boldsymbol{r}_{i}\right), M_{2}\right]} \phi(M) d M / \int_{M_{1}}^{M_{2}} \phi(M) d M .
$$

Here $M_{1}\left(\boldsymbol{r}_{i}\right)$ denotes the minimum and $\boldsymbol{M}_{2}\left(\boldsymbol{r}_{i}\right)$ the maximum absolute magnitude observable at the distance $r$, given the apparent magnitude limits of the field of galaxy $i$, and $\phi(M)$ is the LCRS luminosity function. For $\phi(M)$ we use a Schechter luminosity function (Schechter 1976) with parameters $M^{*}=-20.3+5 \log h$ and $\alpha=-0.7$; details concerning the derivation of the luminosity function are given in Lin et al. (1996).

Figure $4 a$ shows the observed convolved $\hat{\widetilde{P}}(k)$ for the full LCRS sample defined above, computed using the methods detailed in $\S 2$. We have also split the full sample into four subsamples by dividing each of the northern and southern data subsets in half by R.A.; see Table 1 for details. The individual $\widetilde{P}(k)$ for the four subsamples are also plotted in Figure 4. We use the standard deviations of the mean of $\hat{\widetilde{P}}(k)$ for the subsamples, or the $1 \sigma$ errors from the wholeLCRS ODM mock surveys of $\S 2.2$, whichever is larger, to estimate the $1 \sigma$ errors for the full sample. We also note that though we average $\widehat{\widetilde{P}}(\boldsymbol{k})$ over different directions of $|\boldsymbol{k}|$ at fixed $|\boldsymbol{k}|=k$ in equation (14), results from the mock surveys show that the errors depend only weakly on direction. The convolution effects are quite similar for all five samples shown in Figure $4 a$, so that the convolved power spectra may be directly compared. There is good agreement among the four subsamples for wavelengths $\lambda \lesssim 100 h^{-1} \mathrm{Mpc}$. On larger scales, aside from sampling fluctuations, uncertainties in the survey's selection function and mean density will also

TABLE 1

MAGNitUde-Limited SAMPLES

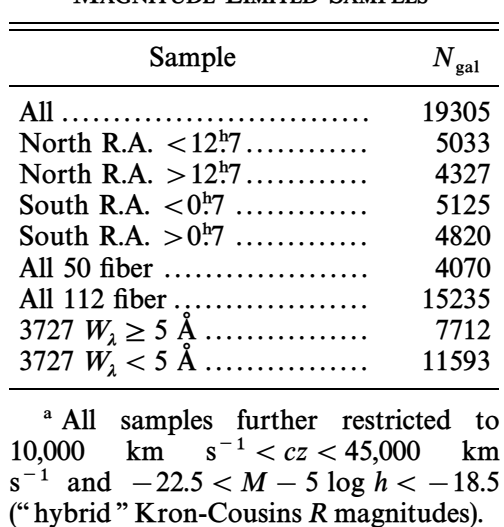
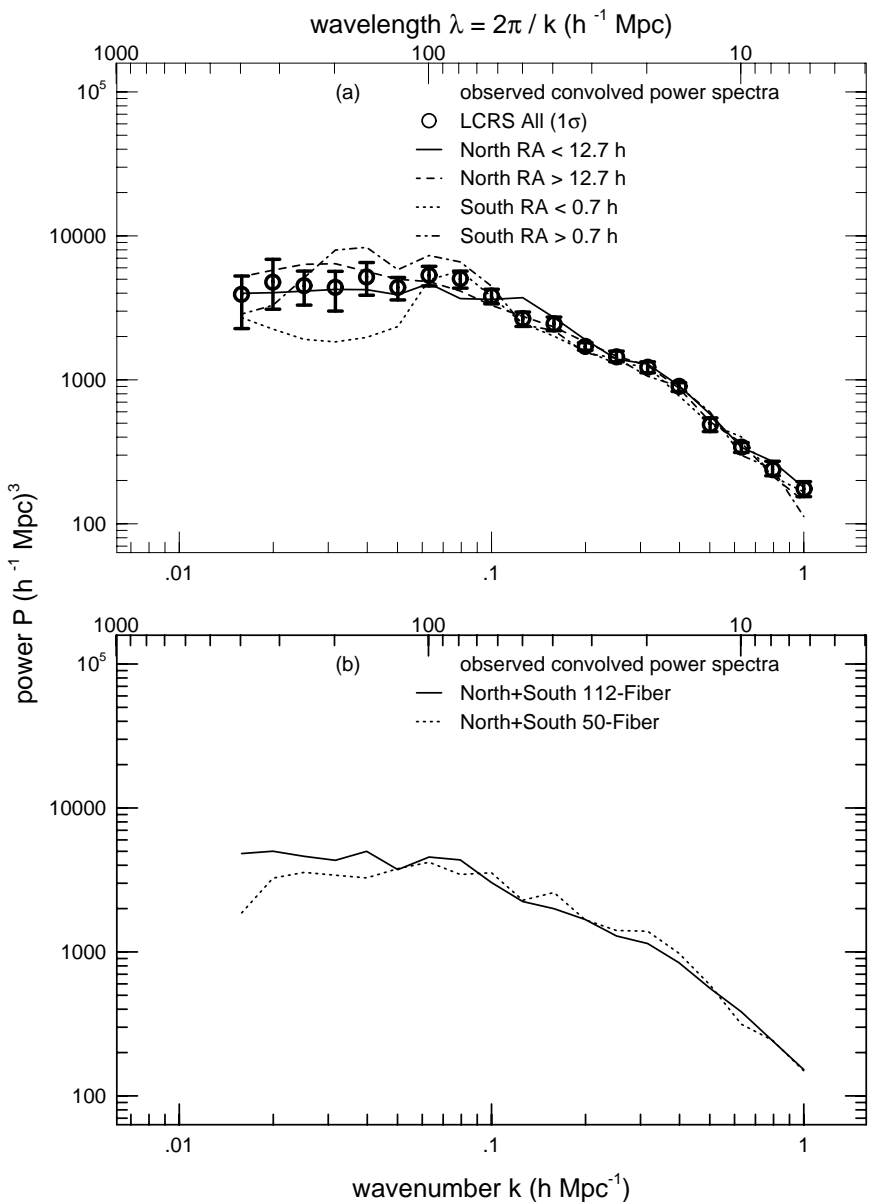

FIG. 4.- (a) Directly measured convolved power spectrum for the full magnitude-limited LCRS sample and for each of the four subsets of the full sample divided by hemisphere and right ascension. (b) The convolved power spectrum for the 112 and 50 fiber subsets of the full sample.

contribute to the differences among the samples. We have also divided the data into 50 fiber and 112 fiber subsets (see Table 1), and computed the convolved power spectrum for each subsample, with the results plotted in Figure 4b. Again, the convolution effects for these two subsets are similar, so we directly compare them. There are no conspicuous systematic differences between the power spectra computed from 50 versus 112 fiber data, giving us confidence that the power spectrum is not sensitive to the different sampling and photometric selection criteria involved. The two subsamples agree well for $\lambda \lesssim 150 h^{-1}$ Mpc.

We can also check the consequences on the power spectrum arising from two geometric selection effects of the survey. First, recall from $\S 1$ that an instrumental constraint prevents two galaxy fibers from approaching closer than $55^{\prime \prime}$ on the sky. In the ODM mock surveys we have actually applied this restriction and our previous results indicate apparently little effect on the power spectrum. More explicitly, we can assign fake velocities to those unobserved objects that lie within $55^{\prime \prime}$ of an observed survey galaxy, and then recompute the power spectrum. Some fraction of these pairs will be physically associated, and we assign velocities to the unobserved objects according to the distribution of pairwise velocity differences for observed survey galaxy pairs with angular separations between $1^{\prime}$ and $2^{\prime}$. For this purpose we use a very generous upper bound of $5000 \mathrm{~km}$ 
$\mathrm{s}^{-1}$ for the velocity difference, corresponding to $60 \%$ of the observed velocity difference distribution. The remaining $40 \%$ of pairs are assumed to be chance superpositions, for which we assign velocities to the unobserved objects according to the overall redshift histogram of the particular survey field containing that pair. Note that this procedure adds an average of 1351 galaxies to the full LCRS sample used for power spectrum computation. Figure $5 a$ plots the full range of the power spectrum computed from 10 different sets of such velocity assignments and confirms that the full-sample LCRS power spectrum is indeed very little affected over the scales probed ( $<10 \%$ difference). Second, as described in Shectman et al. (1995), the spectroscopic success rate is lower (though still more than 90\%) at the corners of the spectroscopic fields. We can approximately compensate for this effect by calculating a "neighborhood" sampling fraction within an area of radius 1000 " centered on each galaxy and then applying this additional geometric weighting in equation (21). The choice of regions of $1000^{\prime \prime}$ radius to define the weights came from trial-and-error efforts to best reproduce the angular pair distribution of the survey photometric catalog, by appropriately weighting the pair distribution of the spectroscopic sample. Figure $5 b$ shows that applying this correction also has little effect on the LCRS power spectrum. For simplicity, since both the

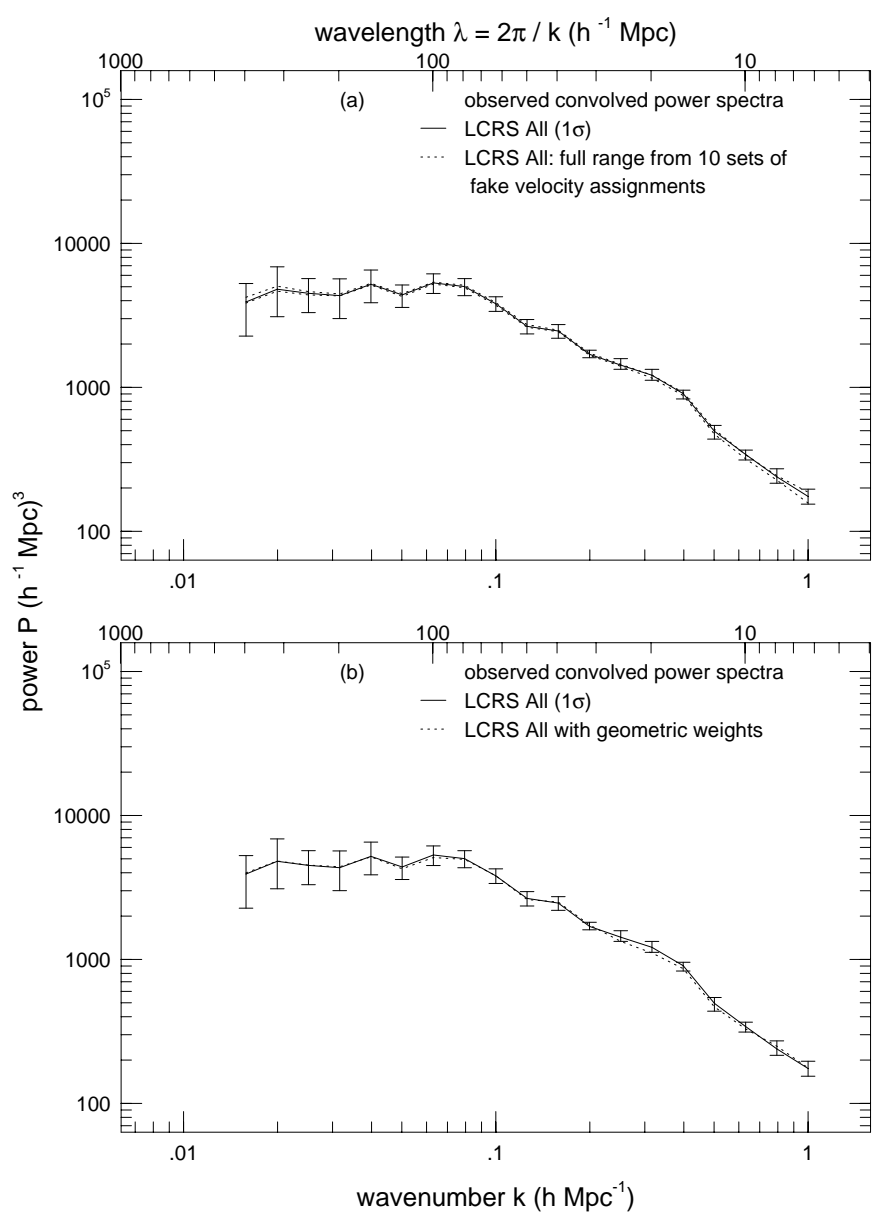

FIG. 5.-Effects on the power spectrum of the full magnitude-limited LCRS sample arising from: $(a)$ assigning fake velocities to unobserved objects lying within $55^{\prime \prime}$ of an observed galaxy; and $(b)$ applying geometric weights to correct for the reduced spectroscopic success rate at field corners. See text for details. above geometric effects are small, we will neglect them in the remainder of this paper.

We next use the Lucy deconvolution procedures outlined in $\S 2$ to obtain estimates of the deconvolved $\hat{P}(k)$ for the full sample and the four subsamples, with the results for the full sample displayed in Figure 6. Here we again estimate errors using the sdom of the four subsamples, or the $1 \sigma$ errors from the deconvolved power spectra of the whole-LCRS ODM mock surveys, whichever is larger. Besides using Lucy deconvolution, we have also convolved a convenient model for the power spectrum and fit to the observed LCRS full sample $\tilde{\tilde{P}}(k)$ :

$$
P(k)=\frac{2 \pi^{2}}{k^{3}} \frac{\left(k / k_{0}\right)^{n_{0}}}{1+\left(k_{c} / k\right)^{n_{c}}} .
$$

The model was motivated by a similar fitting formula applied to the APM data by Peacock (1992) (see also Feldman et al. 1994). The model has four free parameters $\left(k_{0}, n_{0}, k_{c}, n_{c}\right)$, and essentially it behaves as a power law with slope $n_{0}-3$ at small scales (large $k$ ), changing to another power law with slope $n_{0}-3+n_{c}$ at large scales (small $k$ ) (we take $n_{0}, n_{c}>0$ ). The transition occurs near wavenumber $k_{c}$, and there remains an overall normalization that can be more meaningfully expressed in terms of $\sigma_{8}$. We find

$$
\begin{gathered}
k_{c}=(0.06 \pm 0.01) h \mathrm{Mpc}^{-1}, \\
P(k) \propto k^{n_{0}-3}=k^{-1.8 \pm 0.1}, \quad 2 \pi / k=5-30 h^{-1} \mathrm{Mpc}, \\
P(k) \propto k^{n_{0}-3+n_{c}}=k^{1 \pm 1}, \quad 2 \pi / k \approx 200-400 h^{-1} \mathrm{Mpc}, \\
k_{0}=(0.17 \pm 0.01) h \mathrm{Mpc}^{-1} \\
\sigma_{8}=1.0 \pm 0.1 \text { (redshift space) }
\end{gathered}
$$

We plot the fit in Figure 6. Note that for $\lambda \lesssim 100 h^{-1} \mathrm{Mpc}$, the power spectrum is well determined. There is an apparent peak at about $2 \pi / k_{c}=100 h^{-1} \mathrm{Mpc}$, but on larger scales the form of $P(k)$ is not too well determined, with our fit power-law slope consistent with a flat to declining $P(k)$ with decreasing $k$. We want to emphasize that for $\lambda \gtrsim 100$ $h^{-1} \mathrm{Mpc}$, the errors are unavoidably large and we do not claim detection of a turnover in the power spectrum, only that our results suggest that $P(k)$ is at least flattening out on these largest scales. Improved power spectrum estimation techniques, as recently described by Tegmark (1995) or by Vogeley \& Szalay (1996), may prove to be helpful in reducing the errors on the large-scale power spectrum, though application of these methods is beyond the scope of the present paper.

As we mentioned in $\S 1$, a complementary analysis of the two-dimensional LCRS power spectrum may be found in Landy et al. (1996). The wavevectors in the two-dimensional treatment are aligned along the "planes" of each of the six slices, rather than averaged over the whole sphere as they are in this paper. The two-dimensional analysis thus emphasizes those spatial directions for which the survey geometry is best suited on large scales. This renders the two-dimensional analysis less sensitive to the presence of aliased power and therefore more sensitive to the presence of features in the power spectrum on large scales. In particular, a striking peak at $\lambda \approx 100 h^{-1} \mathrm{Mpc}$ is seen in the twodimensional power spectrum, and it is a signature of the clear pattern of over- and underdense regions (e.g., walls 


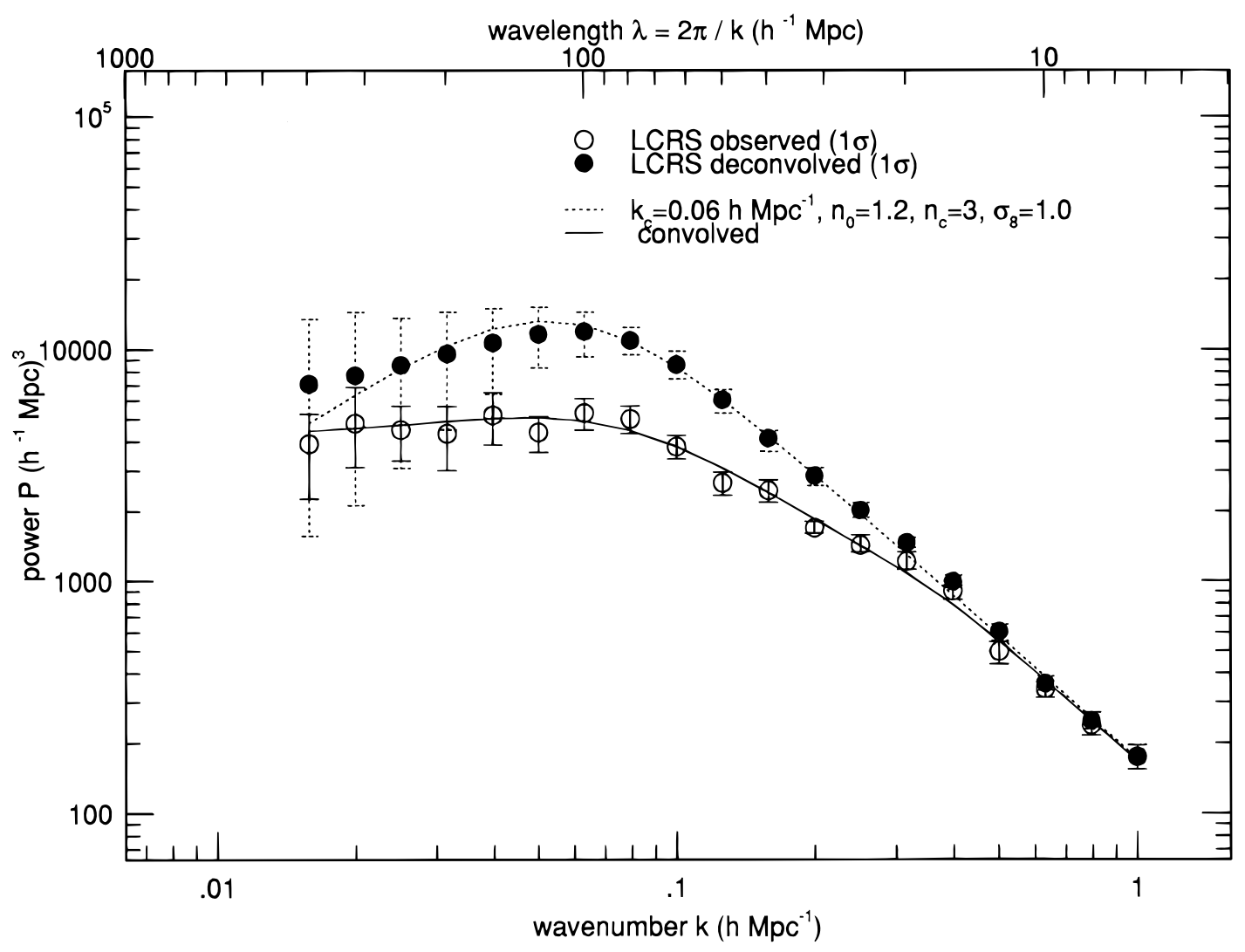

FIG. 6.-Fit to the LCRS power spectrum using the model of eq. (23)

and voids) in the LCRS galaxy distribution (Fig. 1). A different "core-sampling" analysis applied to the LCRS by Doroshkevich et al. (1996) also indicates that $100 h^{-1} \mathrm{Mpc}$ is approximately the typical separation between large sheetlike structures in the galaxy distribution. These results are also reminiscent of a similar peak at $128 h^{-1} \mathrm{Mpc}$ in the one-dimensional power spectrum of deep pencil beam surveys analyzed by Broadhurst et al. (1990). A peak at $\lambda \approx 100 h^{-1} \mathrm{Mpc}$ is also suggested in the three-dimensional power spectrum fit above, though it does not stand out in Figure 6, both because of the logarithmic $y$-axis scaling and because of the suppression due to aliasing as mentioned above. The focus of the two-dimensional paper is on the $100 h^{-1} \mathrm{Mpc}$ scale power, and the reader is referred to Landy et al. (1996) for the full details. On the other hand, the current analysis has the broader goal of relating the full LCRS power spectrum, in the more usual threedimensional context, to results from previous surveys, to $C O B E$ constraints, and to predictions of cosmological models.

\subsection{Comparison to Other Surveys}

We proceed now to compare the LCRS power spectrum to that determined from several other large galaxy redshift surveys. First, Figure 7 shows the comparison to the power spectra of three samples drawn from the SSRS2 and CfA2 redshift surveys: two volume-limited samples SSRS2 + CfA2 $101\left(r<101.05 h^{-1} \mathrm{Mpc}, M_{B}<-19.7+5\right.$ $\log h)$ and SSRS2 + CfA2 $130\left(r<130.0 h^{-1} \mathrm{Mpc}, M_{B}<\right.$ $-20.3+5 \log h$; da Costa et al. 1994b), and one apparentmagnitude-limited sample CfA $101 \mathrm{~m}\left(r<101.05 h^{-1} \mathrm{Mpc}\right.$, $m_{B(0)}<15.5$; Park et al. 1994). Now, while the Las Cam- panas galaxies have been selected from photometry in a red wavelength band, the SSRS2 and CfA2 galaxies were selected in the blue. As a rough guide to compare the red and blue magnitudes, we note that $M_{\text {LCRS }}^{*}=-20.3$ (Lin et al. 1996), while $M_{\mathrm{CfA} 2}^{*}=-18.8$ (Marzke et al. 1994) and $M_{\text {SSRS2 }}^{*}=-19.5$ (da Costa et al. 1994a). Nonetheless, the LCRS $P(k)$ is in remarkably good agreement with the $P(k)$ of the magnitude-limited CfA $101 \mathrm{~m}$ and volume-limited SSRS2 + CfA2 101 samples. There are some differences, though they are not at high significance given the errors. Specifically, the LCRS power spectrum is lower than SSRS2 + CfA2 101 on intermediate scales $\lambda \sim 20-40 h^{-1}$ $\mathrm{Mpc}$, and it is also low relative to these other two samples on large scales $\lambda \gtrsim 150 h^{-1} \mathrm{Mpc}$. In contrast, the power spectrum of the volume-limited SSRS2 + CfA2 130 sample is higher than that of the LCRS on all scales examined. Most simply, these $P(k)$ differences may just be due to cosmic variance that arises from the different volumes being sampled in the three surveys. Alternatively, luminosity bias might help reconcile some of the differences we see, in the sense that intrinsically brighter galaxies are more strongly clustered. We note that the two volume-limited SSRS2 + CfA2 samples are restricted to galaxies brighter than $M_{\text {CfA2 }}^{*}$ and $M_{\mathrm{SSRS} 2}^{*}$, whereas the LCRS sample contains galaxies between approximately $M_{\text {LCRS }}^{*}+2$ and $M_{\text {LCRS }}^{*}-2$. The magnitude-limited CfA $101 \mathrm{~m}$ sample does contain galaxies both brighter and fainter than $M_{\mathrm{CfA} 2}^{*}$ and seems the best match to the LCRS. Park et al. (1994) find evidence for luminosity bias in the CfA survey, and we explore this possibility for the LCRS in $\S 4$. As another alternative, the differences between the two surveys may reflect intrinsic clustering differences of galaxies selected in different wave- 


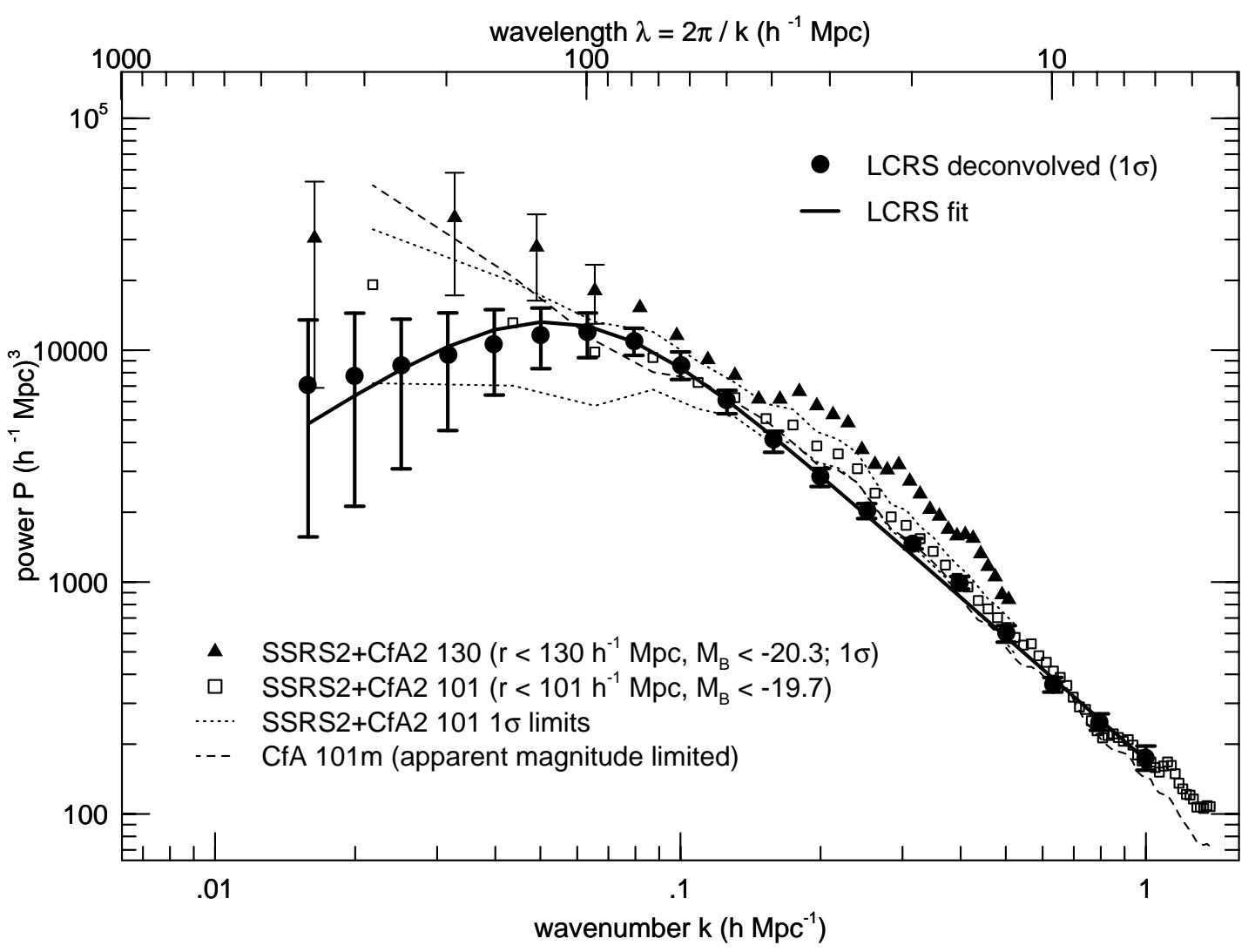

FIG. 7.-Deconvolved LCRS power spectrum and fit compared to the power spectra of three samples drawn from the combined SSRS2 and CfA2 redshift surveys.

length bands, red for the LCRS versus blue for the other two surveys, analogous to the effect observed for CfA galaxies versus infrared-selected IRAS galaxies (see below). The galaxy sampling and surface brightness selection criteria of the LCRS are unlikely to produce a systematic bias in our power spectrum estimates over the scales probed, given the results of the test done in $\S 2.2$ and the agreement of the 50 and 112 fiber results in Figure $4 b$.

We next compare the LCRS $P(k)$ to that found from two redshift surveys of IRAS galaxies: the $1.2 \mathrm{Jy}$ survey $(60 \mu \mathrm{m}$ flux $<1.2 \mathrm{Jy}$; Fisher et al. 1993) and the deeper but more sparsely sampled QDOT sample $(60 \mu \mathrm{m}$ flux $<0.6 \mathrm{Jy}$; Feldman et al. 1994). As seen in Figure 8, the IRAS $1.2 \mathrm{Jy}$ $P(k)$ is lower than that of the LCRS over all scales probed, particularly for $\lambda<30 h^{-1}$ Mpc. Fisher et al. (1993) have shown that the $1.2 \mathrm{Jy} P(k)$ is very similar in shape to the CfA $101 P(k)$, but that the overall clustering amplitude for IRAS galaxies is lower. In redshift space $\sigma_{8}(1.2 J y)=0.8, \sigma_{8}$ (CfA 101) $=1.1$, while the LCRS is in between, with $\sigma_{8}(\mathrm{LCRS})=$ 1.0. The clustering strength seen in the $1.2 \mathrm{Jy}$ survey may reflect the inherently weaker clustering amplitude of the IRAS galaxy population, which tend to be spirals that avoid rich clusters (e.g., Strauss et al. 1992). We have selected from the LCRS a sample of emission galaxies, with [O II] $\lambda 3727$ equivalent widths $W_{\lambda} \geq 5 \AA$, which should also tend to be spirals, as well as a sample of galaxies with $W_{\lambda}<5 \AA$ A, which should include more ellipticals (see Table 1). We compare the relative convolved power spectra of the whole, emission, and nonemission LCRS samples in Figure 9. We find that for scales $\lambda \approx 10-100 h^{-1} \mathrm{Mpc}$, the emission sample is about $30 \%$ more weakly clustered than the full sample, while the nonemission sample is about $25 \%$ more strongly clustered than the full sample, and these results are roughly independent of scale. The same trends persist up to $\lambda \approx 300 h^{-1} \mathrm{Mpc}$, though the errors become larger for $\lambda \gtrsim$ $100 h^{-1} \mathrm{Mpc}$ (here again the errors have been estimated from the variation among the four LCRS subsamples divided by hemisphere and right ascension). Though the difference is not as great as for IR $A S$ versus LCRS galaxies, our results are consistent with an intrinsically weaker clustering strength of spiral and emission-line galaxies on scales $\lambda \lesssim 100 h^{-1} \mathrm{Mpc}$. Galaxies with early morphological types have been observed to be more strongly clustered than late types in a fair number of previous smaller samples, exploring generally smaller clustering scales (e.g., Davis \& Geller 1976; Mo et al. 1992; Loveday et al. 1995). Our current results are thus in qualitative agreement, though the lack of one-to-one correspondence between morphologies and emission strengths vitiates a more quantitative comparison.

We note next from Figure 8 that the LCRS and QDOT results are in somewhat better agreement, except that the QDOT slope is steeper, in the sense of having more relative power for scales $\lambda \sim 100-200 h^{-1} \mathrm{Mpc}$ compared to scales $\lambda \sim 50 h^{-1}$ Mpc. Feldman et al. (1994) also point out a turnover in the QDOT $P(k)$ for $\lambda \gtrsim 150 h^{-1} \mathrm{Mpc}$. The LCRS $P(k)$ shows an apparent peak at roughly similar scales, $\lambda \approx 100 h^{-1} \mathrm{Mpc}$, but the constraint on the slope of the LCRS power spectrum on larger scales is not strong, since it can accommodate both declining to flat power spectra for $\lambda \gtrsim 200 h^{-1} \mathrm{Mpc}(\S 3.1)$. Note finally that the QDOT and 1.2 Jy results differ somewhat for $\lambda>100 h^{-1}$ Mpc. Feldman et al. (1994) attribute this to sampling fluc- 


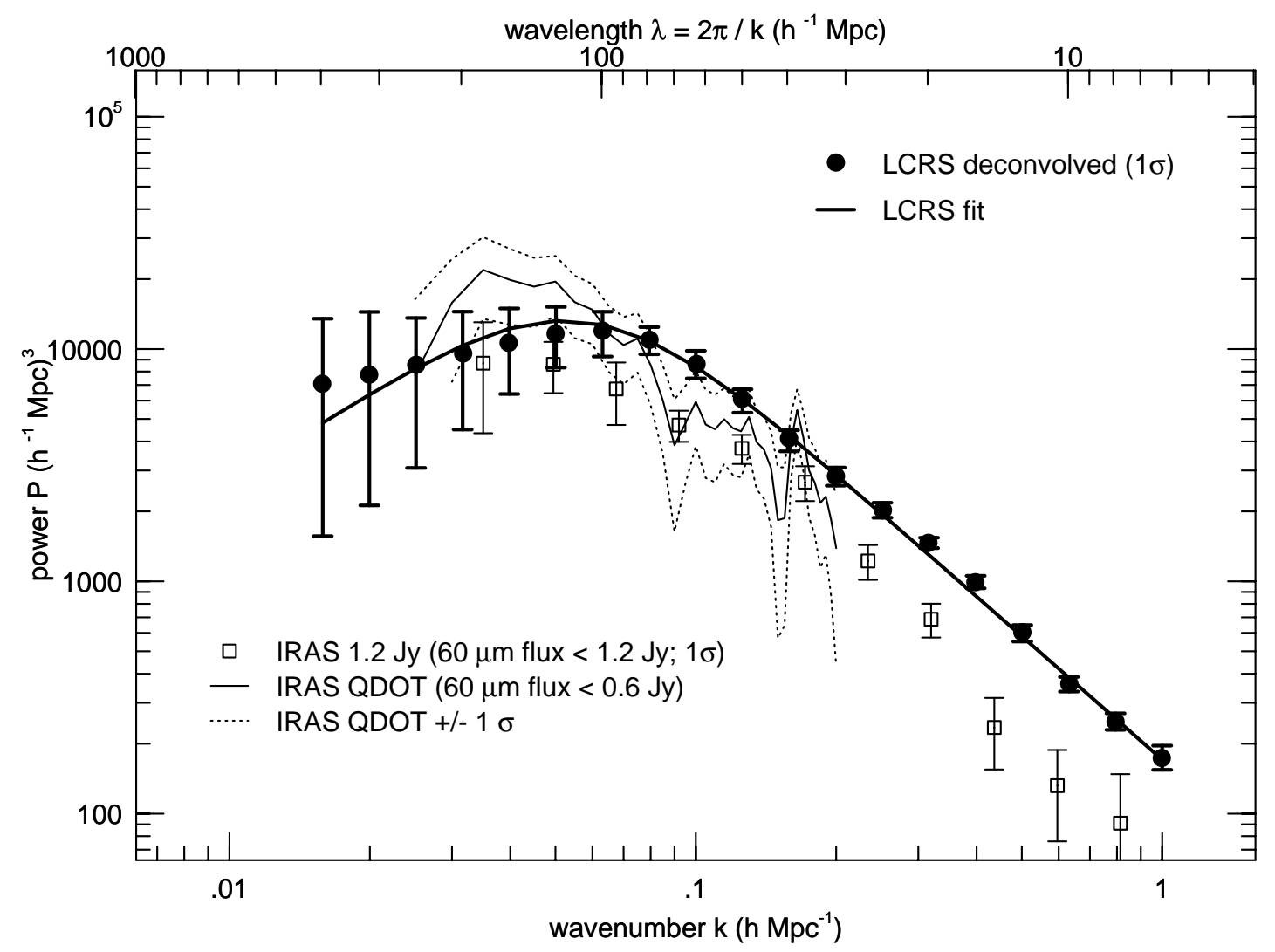

FIG. 8.-Deconvolved LCRS power spectrum and fit compared to the power spectra of the IRAS 1.2 Jy and IRAS QDOT redshift surveys

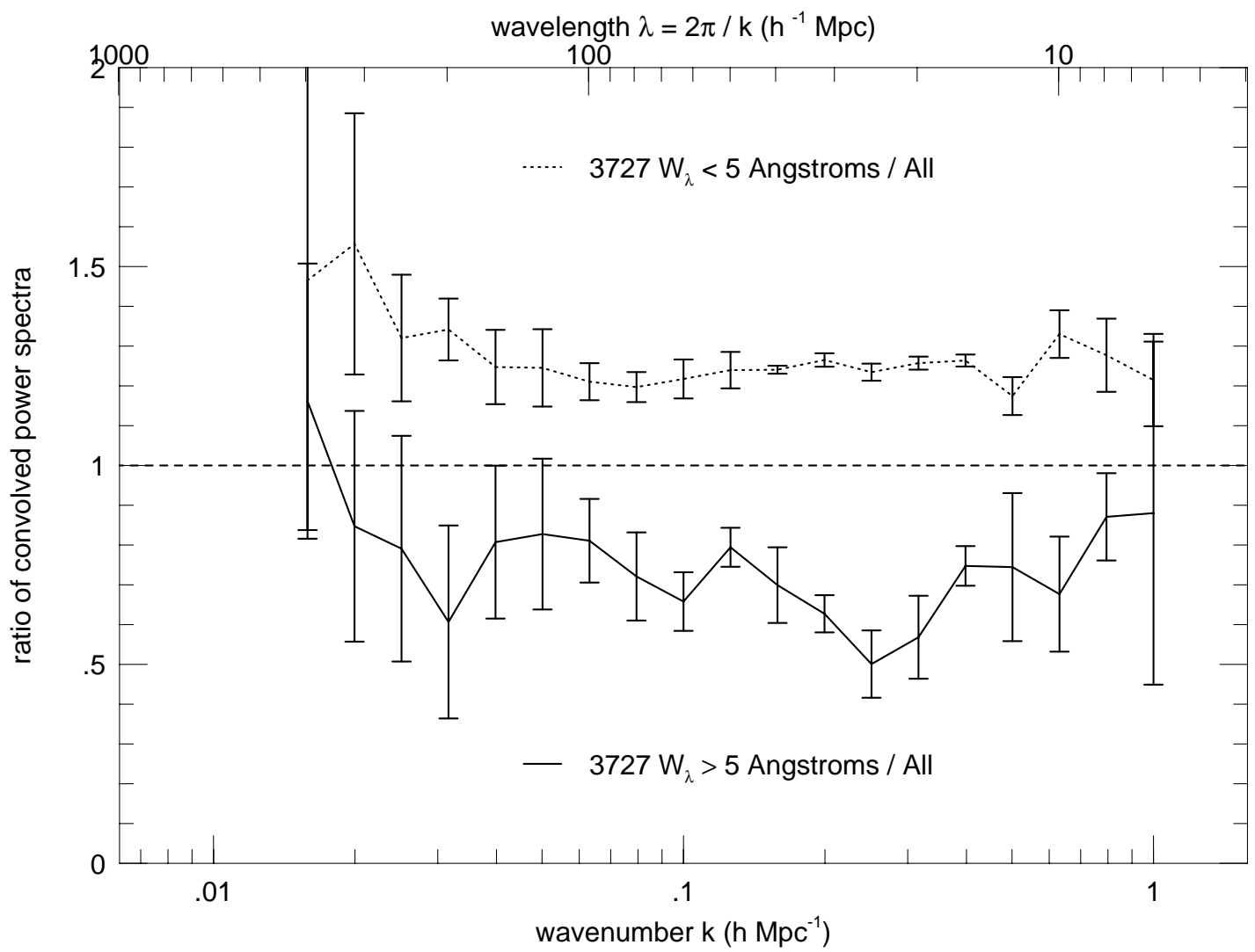

FIG. 9.-Convolved power spectra of two LCRS subsamples, defined by [O II] $\lambda 3727$ equivalent width $W_{\lambda}$, relative to that of the full sample. The errors shown are standard deviations of the mean determined from the four LCRS subsets divided by hemisphere and right ascension. 
tuations rather than to luminosity bias among IRAS galaxies (see also Mann, Saunders, \& Taylor 1996). The sampling fluctuation argument is also indicated by the analysis of Tadros \& Efstathiou (1995), who have calculated the power spectrum of the combined 1.2 Jy and QDOT samples.

Overall, for scales $\lambda \gtrsim 100 h^{-1} \mathrm{Mpc}$, despite some apparent differences, the power spectra from the various surveys are broadly consistent with each other given the large errors common to all the surveys. On smaller scales, the power spectra of the different surveys show similar shapes, but there are differences in amplitude that may reflect intrinsic variations in clustering strength for galaxies of different luminosities or types. We will explore the dependence of clustering on luminosity for LCRS galaxies next. Nonetheless, it is noteworthy that the independent, optically selected surveys LCRS and SSRS2 + CfA2, which also provide the largest galaxy samples, do in fact agree well over the range of scales probed.

\section{LUMINOSITY BIAS IN THE LCRS}

As we saw in $\S 3.2$ and Figure 7, the SSRS2+CfA2 130 sample $\left(M_{B}<-20.3\right)$, which contains intrinsically brighter galaxies than the SSRS2 + CfA2 101 sample $\left(M_{B}<-19.7\right)$, has a power spectrum amplitude $\approx 40 \%$ greater than that of SSRS2 + CfA2 101 (Park et al. 1994; da Costa et al. 1994b). Motivated by this evidence of luminosity bias, here expressed as the variation of power spectrum amplitude with galaxy luminosity in the SSRS2 and CfA2 surveys, we would like to test for evidence of the same in the LCRS. Recall that these two samples are volume limited, with galaxies brighter than $M_{\mathrm{CfA} 2}^{*}=-18.8$ and $M_{\mathrm{SSR} 2}^{*}=-19.5$, whereas our earlier magnitude-limited LCRS sample contains galaxies both brighter and fainter than $M_{\mathrm{LCRS}}^{*}=$ -20.3 . Volume-limited samples, defined by both a lower and an upper bound on absolute magnitude, are a more natural choice over apparent-magnitude-limited samples for the purpose of examining how the power spectrum changes as a function of absolute luminosity. Also, for volume-limited samples the selection function is simpler to deal with. However, the $1.3 \mathrm{mag}$ band in the isophotal magnitude limits of the 50 fiber data restricts drawing volumelimited samples to inconveniently small distance and absolute magnitude ranges, so we will confine the analysis to the 112 fiber data, which have wider $2.7 \mathrm{mag}$ isophotal limits. Within the same redshift limits $10,000 \mathrm{~km}$ $\mathrm{s}^{-1}<c z<45,000 \mathrm{~km} \mathrm{~s}^{-1}$ as applied to the earlier apparent-magnitude-limited full LCRS sample, we form seven volume-limited samples, with absolute magnitude limits $-18.5>M>-19.5, \quad-19.0>M>-20.0, \ldots$, $-21.5>M>-22.5$. Table 2 summarizes details of these samples. In the power spectrum calculation, we set the weight function for galaxy $i$ to $w\left(\boldsymbol{r}_{i}\right)=W_{i}($ see $\S 3.1)$.

One complication that we note from Table 2 is that the different volume-limited samples do not necessarily overlap much in space with one another, and one might wonder whether any differences seen among the samples may be due to sampling fluctuations rather than to actual luminosity bias. In order to better isolate the luminosity dependence, we adopt a procedure similar to that applied by Hamilton (1988) in examining the variation of the correlation function amplitude with galaxy luminosity in the CfA1 survey. We divide each volume-limited sample in half by absolute magnitude (i.e., the $-19.0>M>-20.0$ would be split into
TABLE 2

VOLUME-Limited SAMPLES

\begin{tabular}{|c|c|c|}
\hline Absolute Magnitude Limits ${ }^{a}$ & $\begin{array}{l}\text { Distance Limits }{ }^{\mathrm{b}} \\
\quad\left(h^{-1} \mathrm{Mpc}\right)\end{array}$ & $N_{\mathrm{gal}}$ \\
\hline$-18.5>M>-19.5$ & $98<r<160$ & 732 \\
\hline$-19.0>M>-20.0 \ldots$ & $98<r<198$ & 1867 \\
\hline$-19.5>M>-20.5 \ldots$ & $119<r<243$ & 3179 \\
\hline$-20.0>M>-21.0$ & $147<r<298$ & 4591 \\
\hline$-20.5>M>-21.5 \ldots$ & $181<r<362$ & 4404 \\
\hline$-21.0>M>-22.0 \ldots$ & $224<r<405$ & 2534 \\
\hline$-21.5>M>-22.5$ & $274<r<405$ & 582 \\
\hline
\end{tabular}

a "Hybrid" Kron-Cousins $R$ magnitudes with $h=1$.

${ }^{\mathrm{b}}$ For nominal 112 fiber apparent magnitude limits $15.0 \leq m<17.7$.

two samples with $-19.0>M>-19.5$ and $-19.5>$ $M>-20.0$ ), calculate the ratio of the power spectra of the brighter and fainter subsets (which are located in the same volume of space), and finally multiply (or divide) these ratios in successive volume-limited samples in order to determine the relative clustering amplitudes as a function of luminosity. We arbitrarily set the clustering amplitude of the $-20.0>M>-20.5$ sample, which contains $M_{\text {LCRS }}^{*}=$ -20.3 , to unity. We apply this procedure to the wholesurvey volume-limited samples and estimate errors from the variation among volume-limited samples from the four subsets of the whole survey divided by hemisphere and right ascension (recall Fig. $4 a$ ). We also average our results over four roughly equal bins in $\log \lambda$, as shown in Figure 10: $\lambda=5-15,20-50,60-150$, and $200-400 h^{-1} \mathrm{Mpc}$. We note first from the figure that results for the bin at $200-400 h^{-1}$ $\mathrm{Mpc}$ are quite noisy and the errors are large enough to be consistent with no variation of clustering amplitude with luminosity. We will focus attention on the three bins in the range from $5-150 h^{-1} \mathrm{Mpc}$, where the errors are not so large. We also find that the variation of clustering amplitude with absolute magnitude is fairly scale-independent over this range of $\lambda$. We see that for $-18.5>M>-21.0$ the clustering amplitude changes little with absolute magnitude. For galaxies brighter than $M=-21.0$, the clustering amplitude appears to rise with luminosity; in particular, for $-21.5>M>-22.0$, the brightest bin with still reasonably small errors, the clustering amplitude is $1.6 \pm 0.5$ that at $M^{*}$. The departures from no luminosity bias are relatively mild, except in the brightest bin, where unfortunately the errors are also large. Nevertheless, there does appear to be about a $50 \%$ stronger clustering for galaxies brighter than about $M^{*}-1$ relative to those fainter, in general agreement with the amount of luminosity bias seen in the CfA2 and SSRS2 surveys. The trends seen in Figure 10 are similar to analogous results for scales $\lesssim 10 h^{-1} \mathrm{Mpc}$ from the much smaller CfA1 survey (Hamilton 1988). Our results are also similar to recent findings from the large-volume, but 1-in-20 sparse-sampled, Stromlo-APM survey (Loveday et al. 1996; Loveday et al. 1995), except that we do not see a much weaker clustering amplitude for sub- $M^{*}$ galaxies that Loveday et al. find. The difference might be due the fact that we do not use galaxies fainter than about $M^{*}+2$ that were included in the analysis of the Stromlo-APM data. In addition, recent correlation function analysis of the SSRS2 sample (Benoist et al. 1996) indicates a luminosity bias trend similar to that seen in the LCRS. We note, however, that the LCRS provides advantages with its combination of sample size, sampling density, and depth over other redshift 

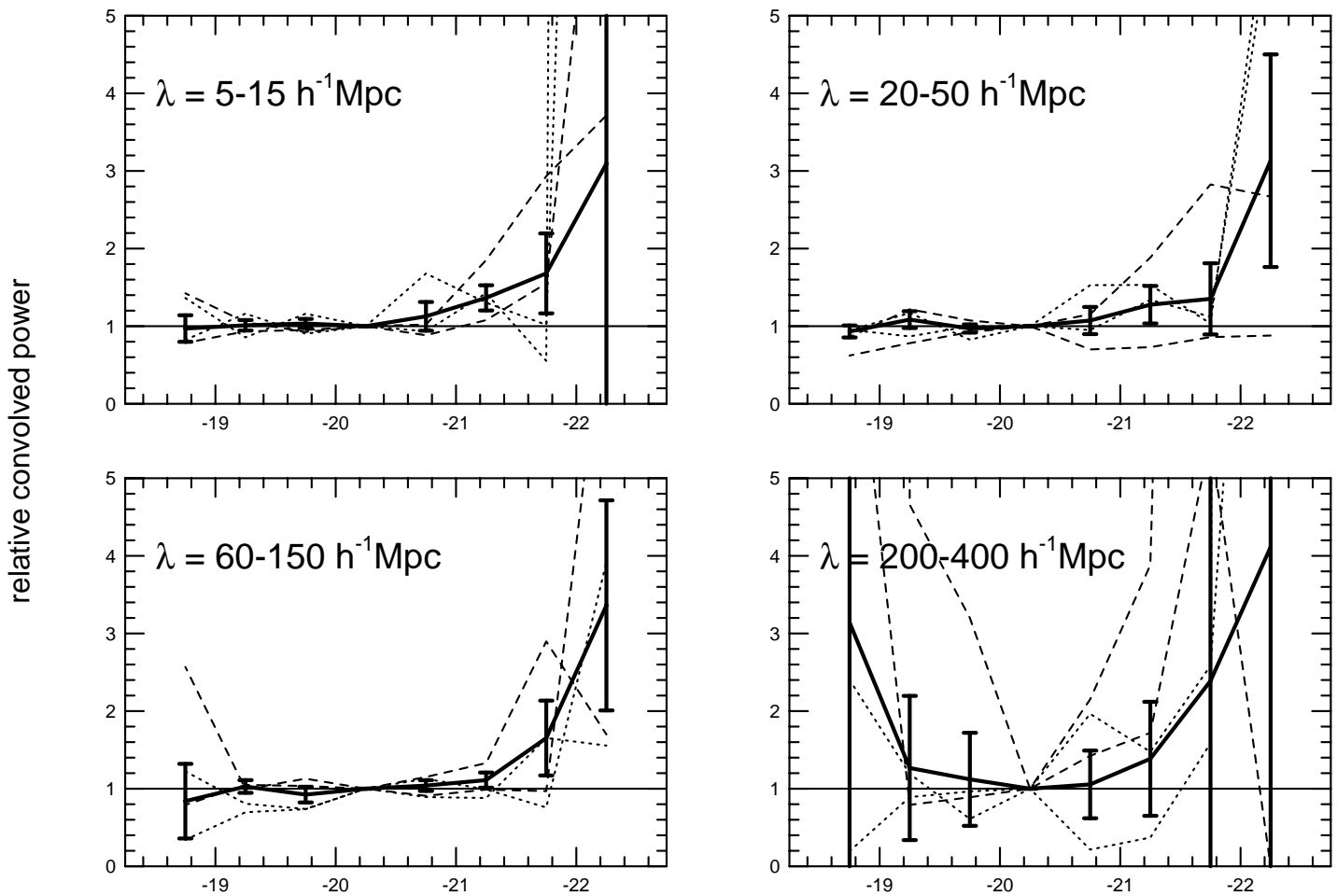

absolute magnitude $\mathrm{M}$

FIG. 10.- Relative convolved power as a function of absolute magnitude, normalized to one at $M=-20.25$, shown for the full LCRS sample (heavy solid lines) and for the four LCRS subsamples divided by hemisphere and right ascension (light broken lines). The errors shown are one standard deviation of the mean from the four subsamples.

surveys, and likely provides the best current sample to examine the luminosity bias effect.

The fact that the luminosity bias we see for super- $M^{*}$ galaxies persists to fairly large scales argues for a primordial rather than a local environmental effect as its root cause. Specifically, our results are qualitatively consistent with the biased galaxy formation scenario, in which brighter galaxies form out of higher peaks in the underlying mass distribution and are therefore more strongly clustered, because such higher peaks are naturally more clustered in models like CDM with an initially Gaussian field of mass density fluctuations (Bardeen et al. 1986; White et al. 1987). Also, the large-scale clustering bias seen earlier for our emission and nonemission samples is also tied to some extent into the luminosity bias issue, as we have found that our nonemission sample is dominated by super- $M^{*}$ galaxies and our emission sample by sub- $M^{*}$ objects (Lin et al. 1996). If the emission ( $\sim$ morphology) and luminosity bias effects are independent, as evidenced in the Stromlo-APM survey (Loveday et al. 1995), then some primordial influence would also be suggested in relation to the galaxy star formation rate, for which [O II] $\lambda 3727$ is a rough indicator (Kennicutt 1992). In the present paper we have simply laid out our initial observations of these type-dependent clustering differences; we plan a more detailed examination of the effects we have found and their quantitative implications for biased galaxy formation models. In addition, note that our present results have been limited to redshift space, and it will be useful, though expectedly more difficult and noisy, to factor out the redshift distortion effects by examining the clustering in real space, which we also plan to attempt.
We can, however, draw some brief conclusions in the context of the recent semianalytic galaxy formation models of Kauffmann, Nusser, \& Steinmetz (1996). In particular, their work indicates that the bias factor $b$ should depend only weakly on scale (also Weinberg 1995), consistent with our observations. Also, their models predict that the amount of luminosity bias should decrease with increasing $\sigma_{8}$ of the underlying mass power spectrum. Specifically, their Figure 6 indicates that on scales $\sim 20 h^{-1} \mathrm{Mpc}$, galaxies with $M \lesssim M^{*}+1$ are more biased relative to those with $M \lesssim M^{*}+3$, by factors of about 1.5 for $\sigma_{8 \text {, mass }}=0.4$ and $\lesssim 1.2$ for $\sigma_{8 \text {, mass }}=1$. For comparison, we can convert the relative power values of Figure 10 into relative bias values (recall power $\propto b^{2}$ ), and then make the appropriate sum over the LCRS luminosity function in order to determine the average bias over some absolute magnitude range. We find for scales $5-150 h^{-1} \mathrm{Mpc}$ that the bias of $M \lesssim M^{*}$ galaxies is less than 1.1 times that of $M \lesssim M^{*}+2$ galaxies (the stronger clustering of bright galaxies is more than compensated by their reduced numbers in the luminosity function). Our results are on the weak luminosity bias end of the range of Kauffmann et al. models and thus imply that $\sigma_{8 \text {, mass }}$ should be high, $\approx 1$. As this is near the observed galaxy $\sigma_{8}$, it also indicates that LCRS galaxies may be unbiased tracers of the mass. We return to this point below in $\S 5.2$.

\section{COMPARISONS WITH COSMOLOGICAL MODELS}

We now compare the LCRS results against the expectations of cosmological models. We begin with a brief comparison using two particular $N$-body simulations, which 
allow us to examine the model power spectra over both small and large scales. Then we proceed to widen the explorable parameter space by concentrating on fitting the LCRS power spectrum on large scales in the linear regime and compare against the predictions of several classes of CDM-motivated models. In combination with $C O B E$ and other large-scale structure constraints, the large-scale LCRS power spectrum will help us delineate the allowed parameter space in these cosmological models.

\subsection{Comparison to $N$-Body Simulations}

We compare the LCRS power spectrum to that of two $N$-body models, both kindly provided by Changbom Park: (1) the ODM model of $\S 2.2$, with $\Omega_{0}=0.4, h=0.5$, and bias factor $b=1$; and (2) the CDM1 model, which has $\Omega_{0}=1$, $h=0.5$, and $b=1.5$. The CDM1 model was computed with a particle-mesh code on a $324^{3}$ mesh, physical comoving volume $\left(388.8 h^{-1} \mathrm{Mpc}\right)^{3}$, and contains $162^{3} \mathrm{CDM}$ particles and 1,201,320 biased "galaxy" particles, chosen by the biasing scheme of Park (1991). Both models are normalized so that the galaxy $\sigma_{8}=1$.

The convolved redshift-space power spectra of the LCRS and the $N$-body models are plotted in Figure 11 . The three power spectra agree well with each other for $\lambda<20 h^{-1}$ Mpc. On intermediate scales from $\lambda \sim 30$ to $50 h^{-1} \mathrm{Mpc}$, the CDM1 model matches the LCRS results somewhat better than the ODM model does. For wavelengths $\lambda>60 h^{-1} \mathrm{Mpc}$, the LCRS power falls in between the CDM1 and ODM curves, with the LCRS results closer to that of CDM1 on the largest scales $\lambda>100 h^{-1}$ Mpc. Though both $N$-body models match the LCRS on small scales, neither model provides quite the right amount of large-scale power: not enough power in the case of CDM1 and too much in the case of ODM.

To be more quantitative in our comparison, we can use a rank sum test to see how probable it is to draw the LCRS power spectrum from the population of $N$-body mock survey power spectra. We choose the rank-sum test as it is simpler to apply than a $\chi^{2}$ test; we need not make any assumptions about either the distribution of power at each $k$ or about the correlations between the power at different $k$. At each wavenumber $k$, we first assign each of the 30 ODM mock survey plus 1 LCRS samples a rank $R_{i, k}$ in order of increasing power (the index $i$ denotes the sample). Then, for each of the 31 samples we combine the ranks at different $k$ by forming the sum $S_{i}=\sum_{k} R_{i, k}$. Finally, we assign each

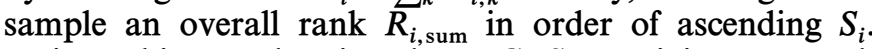
Doing this results in the LCRS receiving a rank $R_{\mathrm{LCRS} \text {, sum }}=2$, giving us a two-tailed confidence interval of $100-2 \times(2 / 31 \times 100)=87 \%$ for rejecting the null hypothesis that the LCRS power spectrum is drawn from the population of ODM power spectra. This is not very high significance, and it will be further weakened by the fact that since we only have one ODM simulation box, we do not sample the full range of variation as we would given an entire ensemble of ODM simulation boxes. Nevertheless, the rank sum test does give us a simple quantitative measure of how well the ODM model can match the LCRS data. Likewise, the rank sum test applied to 30 CDM1 mock survey plus 1 LCRS samples gives $R_{\mathrm{LCRS} \text {, sum }}=10$, where we now assign $R_{i, k}$ in order of decreasing power because the LCRS power is greater than that of most of the

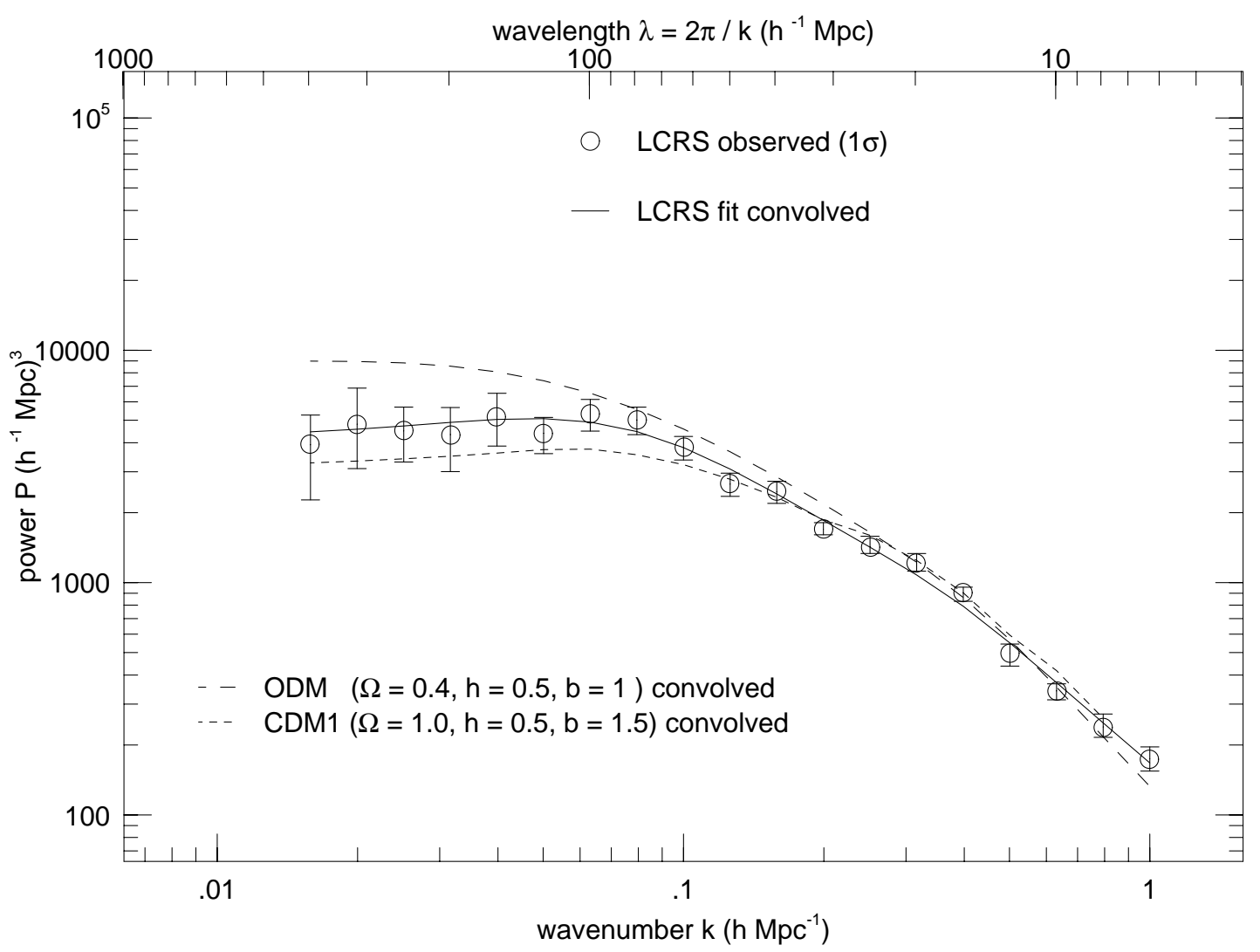

FIG. 11.-Observed convolved LCRS power spectrum compared to the convolved power spectra of the $N$-body models ODM and CDM1. See text for details. 
CDM1 mock surveys. The two-tailed confidence interval for rejecting CDM1 is then just $35 \%$. Repeating the rank sum tests, but now just focusing on large scales $k \leq 0.1 h$ $\mathrm{Mpc}^{-1}$, we find $R_{\mathrm{LCRS} \text {, sum }}=1$ for both ODM and CDM1, so now the rejection probabilities are $94 \%$ in both cases. The match of the CDM1 model to the LCRS is thus appreciably worse when considering only large scales, while the match of the ODM model is little changed. Neither model provides a completely satisfactory match to the LCRS power spectrum over all the scales that we probe. The LCRS power spectrum lies between that of the two models, and as we will see in the next section, the LCRS power spectrum on large scales is better matched by a linear CDM model with $\Omega_{0} h \approx 0.3$.

\subsection{Fitting the Linear Power Spectrum and Comparison to $C O B E$}

For any particular structure formation model, the best comparison procedure with observations is to draw out mock redshift surveys as we did in $\S 2.2$. However, it will be useful to fit our observations to analytic models in order to explore more of cosmological parameter space than permitted by Park's two $N$-body models. To do a proper fit to a nonlinear, redshift space power spectrum given a linear, real space model requires us to account for the following: (1) the nonlinear evolution of the power spectrum; (2) the bias relating the galaxy fluctuations to the underlying mass fluctuations; and (3) the distortions caused by mapping from real to redshift space. Though these three effects may be modeled analytically (Peacock \& Dodds 1994; Jain, Mo, \& White 1995), we will take a simpler route and concentrate on fitting just those observations on large scales in, at least approximately, the linear regime. We thus sacrifice the information we have on small scales for the simplicity of not having to model the nonlinear effects (which perhaps are best dealt with via $N$-body simulations).

To specify our assumptions, note the following relations connecting the galaxy power spectrum and the underlying mass power spectrum:

$$
\begin{gathered}
P_{\text {galaxy, real space }}(k)=f_{b}(k) P_{\text {mass, real space }}(k), \\
P_{\text {galaxy,redshift space }}(k)=f_{z}(k) P_{\text {galaxy, real space }}(k),
\end{gathered}
$$

where $f_{b}(k)$ is some function describing the bias of the galaxy power spectrum relative to that of the mass, and $f_{z}(k)$ describes the effect of redshift distortions caused by galaxy peculiar velocities. In general, the particular functional forms of $f_{b}$ and $f_{z}$ will depend on the parameters of the cosmological model. We make the simple assumption that over the scales we consider, $f_{b}(k)=b^{2}=$ constant, where $b$ is the bias factor. For $f_{z}(k)$, there are two main regimes. As derived by Kaiser (1987), on large scales in the linear regime, the redshift space power spectrum is amplified by the infall of galaxies from low-density regions to highdensity regions, and

$$
f_{z}(k) \approx 1+\frac{2}{3} \frac{\Omega_{0}^{4 / 7}}{b}+\frac{1}{5} \frac{\Omega_{0}^{8 / 7}}{b^{2}} .
$$

(More commonly in the literature the fractions 4/7 and 8/7 in the above equation are replaced by 0.6 and 1.2 , respectively; but see Lightman \& Schechter 1990.) On the other hand, on small scales in the nonlinear regime, the redshift space power spectrum is suppressed by the smearing of the clustering pattern from small-scale galaxy peculiar veloci- ties. For example, if we assume that galaxies have a smallscale Gaussian peculiar velocity field with a one-dimensional dispersion $\sigma_{v}$, then

$$
f_{z}(k)=\frac{\sqrt{\pi}}{2} \frac{\operatorname{erf}\left(k \sigma_{v} / H_{0}\right)}{k \sigma_{v} / H_{0}}
$$

(Peacock 1992). However, here we will focus on the linear regime and so assume that

$$
\begin{aligned}
& P_{\text {galaxy, redshift space }}(k) \\
& \quad \approx b^{2}\left[1+\frac{2}{3} \frac{\Omega_{0}^{4 / 7}}{b}+\frac{1}{5} \frac{\Omega_{0}^{8 / 7}}{b^{2}}\right] P_{\text {mass, real space,linear }}(k)
\end{aligned}
$$

holds as a useful approximation, that is, the redshift space galaxy power spectrum is just a constant times the real space mass power spectrum.

Since the density fluctuations in the galaxy distribution should be less than unity in the linear regime, and since we observe that the galaxy fluctuations in $8 h^{-1} \mathrm{Mpc}$ radius spheres $\sigma_{8}=1$, we need to restrict our fits at least to $k<2 \pi /$ (several $\times 10 h^{-1} \mathrm{Mpc}$ ). We will use $k \leq 0.16 h$ $\mathrm{Mpc}^{-1}$, corresponding to $\lambda \geq 40 h^{-1} \mathrm{Mpc}$. Though this choice is somewhat arbitrary, it is motivated by our desire to extend the fit range beyond the flat part of the convolved power spectrum (to improve the handle on the power spectrum shape), but not so far that nonlinear effects become important. That equation (29) approximately holds over our $k$ range has justification. Theoretical work (e.g., Kauffmann et al. 1996; Weinberg 1995) indicates that constant linear biasing does hold on large scales. There is also empirical evidence, from the comparison of real and redshift space galaxy power spectra from the survey compilation of Peacock \& Dodds (1994), and in particular, from the comparison of various galaxy survey power spectra (including LCRS) against the mass power spectrum derived from peculiar velocity catalogs over the range $0.05 \leq k \leq 0.2 h$ $\mathrm{Mpc}^{-1}$ (Kolatt \& Dekel 1996). We will thus fit various model linear power spectra (to be described below) to the full sample magnitude-limited LCRS convolved power spectrum for $0.016 \leq k \leq 0.16 h \mathrm{Mpc}^{-1}$. One complication is that because of correlations among the data points on large scales, a rigorous fit requires us to calculate and account for the covariance matrix of the errors (e.g., Feldman et al. 1994). However, for simplicity we take a more empirical approach and estimate the errors for the fit parameters from the variation among our usual four LCRS subsamples and from the $N$-body mock surveys.

We focus on the case of inflationary, dark-matter dominated cosmological models, in which case we may write

$$
P_{\text {mass, real space, linear }}(k)=B_{\text {mass }} k^{n} T^{2}(k),
$$

where $B_{\text {mass }}$ is a normalization factor, $k^{n}$ is the inflationproduced primordial power spectrum of spectral index $n$, and $T(k)$ is a transfer function that modifies the primordial spectrum. Note that the shape of the power spectrum is parameterized by the index $n$ and by the form of $T(k)$, which in turn depends on the specific matter content of the universe. Now because we are fitting over a relatively small range in $k$, and because the convolved power spectrum tends to flatten out over the same $k$ range, regardless of the true power spectrum shape, the LCRS results will not constrain the true shape very strongly. On the other hand, given a specific power spectrum shape, the LCRS results do 
give fairly stringent constraints on the power spectrum normalization. Using our earlier mock surveys and the usual procedure of dividing into four subsamples, we estimate a 1 $\sigma$ fractional error of about $10 \%$ in the normalization, obtained by fitting to the full-sample LCRS power spectrum over our desired $k$ range. However, there is still freedom in the choice of bias parameter $b$ (eq. [27]) so that we cannot fix an absolute normalization from our galaxy data. The absolute mass normalization $B_{\text {mass }}$ can instead be determined independently using results from the COBE DMR experiment. We can then turn the problem around and use our derived galaxy normalization $B_{\mathrm{LCRS}}$ to determine the value of $b$ needed to reconcile the LCRS and COBE normalizations via the relation

$$
b^{2}\left(1+\frac{2}{3} \frac{\Omega_{0}^{4 / 7}}{b}+\frac{1}{5} \frac{\Omega_{0}^{8 / 7}}{b^{2}}\right) B_{\text {mass }} \approx B_{\mathrm{LCRS}} .
$$

We note that theoretical models suggest a bias $b$ near unity or larger (e.g., Kauffmann et al. 1996). Empirically, we see within the LCRS that emission/nonemission samples have bias values within $\approx \pm 15 \%$ that of the full sample (power $\propto b^{2}$ ), and that super- $\bar{M}^{*}$ galaxies have bias $\approx 1.3$ times that of fainter galaxies. Likewise, Peacock \& Dodds (1994) find a relative bias between optical and IRAS galaxies of $b_{\text {optical }}: b_{I R A S}=1.3$, and Kolatt \& Dekel (1996) find a range 0.77-1.21 in relative bias parameters for various surveys, including LCRS. In particular, the latter authors find $b_{\mathrm{LCRS}}=\Omega_{0}^{0.6} /(0.99 \pm 0.13)$ for $k=0.05-0.2 h \mathrm{Mpc}^{-1}$. For $\Omega_{0}=1$, this corroborates the earlier indication from our luminosity bias results that LCRS galaxies may have $b \approx 1$. In any event, it appears that 0.7-1.3 may represent a reasonable range in (absolute) bias values for galaxies, and the assumption that LCRS galaxies are roughly unbiased should serve as a useful guide to help constrain the models.

To narrow down the cosmological parameter space to be examined, we will consider four simple variants of the basic flat, inflationary CDM model with $\Omega_{0}=1$ and $n=1$. These are (1) $\Lambda \mathrm{CDM}$, flat CDM models with nonzero cosmological constant $\Omega_{\Lambda}=1-\Omega_{0}$; (2) OCDM, open CDM models with $\Omega_{0}<1$; (3) CHDM, flat models with both CDM and hot dark matter in the form of massive neutrinos; and (4) TCDM, flat CDM models with a tilted primordial spectrum of index $n \neq 1$. These models were motivated by efforts to address various deficiencies in matching the "standard" $\Omega_{0}=1, h=0.5 \mathrm{CDM}$ model to observational constraints (e.g., see Liddle et al. 1996a, 1996b, 1996c, and references therein). For all these models we need the basic CDM transfer function (Bardeen et al. 1986)

$$
\begin{aligned}
& T_{\mathrm{CDM}}(q)=\frac{\ln (1+2.34 q)}{2.34 q}[ 1+3.89 q+(16.1 q)^{2} \\
&\left.+(5.46 q)^{3}+(6.71 q)^{4}\right]^{-1 / 4},
\end{aligned}
$$

where $q=k / h \Gamma$ and $\Gamma$ is the so-called shape parameter. For no baryonic matter $\Gamma$ is simply $\Omega_{0} h$, but more generally (e.g., Liddle et al. 1996c)

$$
\Gamma=\Omega_{0} h \exp \left(-\Omega_{B}-\Omega_{B} / \Omega_{0}\right),
$$

where $\Omega_{B}$ is the fraction of critical density in baryons. We will adopt the big bang nucleosynthesis value $\Omega_{B}=0.015 / h^{2}$ (Copi, Schramm, \& Turner 1995). As a parenthetical remark, we note that in previous LCRS papers (e.g., Landy et al. 1996; Lin 1995) we used a slightly different CDM transfer function (Bond \& Efstathiou 1984; Efstathiou, Bond, \& White 1992) that has $\Omega_{B}=0.03$ and $\Gamma=\Omega_{0} h$ rather than the current definition (33). The difference in $\Gamma$ is only $6 \%$ for $\Omega_{0}=1$. For our four cases we have

$T(k)= \begin{cases}T_{\mathrm{CDM}}(q) & \Lambda \mathrm{CDM}, \mathrm{OCDM}, \mathrm{TCDM}, \\ T_{\mathrm{CDM}}(q) D\left(q, \Omega_{v}\right) & \mathrm{CHDM},\end{cases}$

where $D\left(q, \Omega_{v}\right)$ is a somewhat complicated function describing the effects of massive neutrinos (it is given in detail in Pogosyan \& Starobinsky 1995 and Liddle et al. 1996b). For our power spectrum definition, the necessary $C O B E$ normalizations may be expressed via

$$
B_{\text {mass }}=2 \pi^{2}\left(c / H_{0}\right)^{3+n} \delta_{H}^{2},
$$

where the 4 year $C O B E$ DMR data (Bennett et al. 1996) give (Liddle et al. 1996b, 1996c; Viana \& Liddle 1996; Bunn \& White, in preparation)

$$
10^{5} \delta_{H}=\left\{\begin{array}{c}
1.94 \Omega_{0}^{-0.785-0.05 \ln \Omega_{0}} \\
\times \exp \left[-0.95(n-1)-0.169(n-1)^{2}\right] \\
\Lambda \operatorname{CDM}, \operatorname{TCDM}, \operatorname{CHDM}\left(\Omega_{0}=1\right) \\
1.95 \Omega_{0}^{-0.35-0.19 \ln \Omega_{0}} \operatorname{OCDM},
\end{array},\right.
$$

where the $1 \sigma$ fractional uncertainty in $\delta_{H}^{2}$ is $15 \%$ (quoted from Liddle et al. 1996b). Also, to provide an additional handle on our parameter space, we consider the constraints provided by the observed abundance of galaxy clusters. The cluster results may be expressed in terms of the (linear theory) value of $\sigma_{8 \text {,mass }}$ as follows (Viana \& Liddle 1996; Liddle et al. 1996b, 1996c):

$\sigma_{8, \text { mass }}= \begin{cases}0.60 \Omega_{0}^{-\left(0.59-0.16 \Omega_{0}+0.06 \Omega_{0}{ }^{2}\right)} & \Lambda \mathrm{CDM}, \mathrm{TCDM}, \\ 0.60 \Omega_{0}^{-\left(0.36+0.31 \Omega_{0}-0.28 \Omega_{0}{ }^{2}\right)} & \mathrm{OCDM} \\ 0.60+0.2 \Omega_{v} / 3 & \mathrm{CHDM}\end{cases}$

where the $95 \%$ confidence uncertainties are $+32 \%$ and $-24 \%$ (but enlarged by the factor $\Omega_{0}^{0.26} \log _{10} \Omega_{0}$ for $\Lambda$ CDM and by $\Omega_{0}^{0.17 \log _{10} \Omega_{0}}$ for OCDM; see Viana \& Liddle 1996).

We consider each of our four classes of CDM models in turn.

$\Lambda C D M$.- - Here we find a shape parameter $\Gamma=0.3 \pm 0.1$ (the error is one sdom among the four subsamples). We plot in Figure $12 a$ the resulting bias $b$ as a function of $\Omega_{0}$, where the error bands are approximate $2 \sigma$ values obtained by adding in quadrature the estimated $2 \sigma$ fractional uncertainties in the LCRS $(20 \%)$ and COBE $(30 \%)$ normalizations $B$. Four sets of curves are shown, drawn for $\Gamma=0.3,0.2$, and for fixed Hubble constants $h=0.5$ and 0.8 . (We note that a variety of measures of $h$, using Cepheids, supernovae, Tully-Fisher distances, surface brightness fluctuations, and other methods, indicate that $h \gtrsim 0.5$, and likely of order $0.6-0.8$; see, e.g., Jacoby et al. 1992 ; Freedman et al. 1994; Schmidt et al. 1994; Riess, Press, \& Kirshner 1995). Recalling our earlier discussion, we also draw horizontal lines at $b=0.7$ and 1.3 to roughly indicate the range of reasonable bias values. In Figure $12 b$ we plot the corresponding $C O B E$-normalized $\sigma_{8 \text {,mass }}$-values $(2 \sigma$ error bands) and the $95 \%$ confidence limits from the cluster abundance constraints. We find that the preferred values $\Gamma=0.3$ and $0.5 \lesssim h \lesssim 0.8$ indicate $0.4 \lesssim \Omega_{0} \lesssim 0.7$, but that these models are antibiased, $b \sim 0.5$, and also violate the cluster abundance constraints. On the other hand, for $\Gamma=0.2$, we do find models at $\Omega_{0} \approx 0.4-0.5$ and $h \approx 0.5$, 


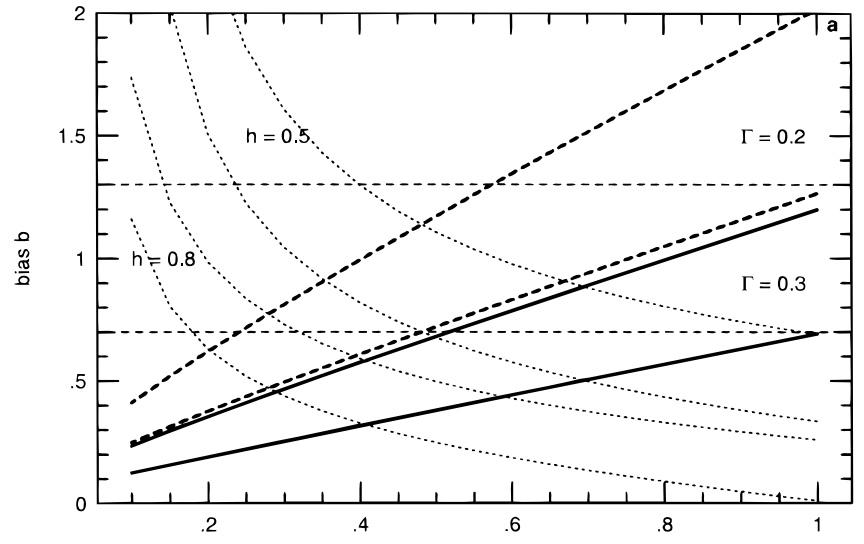

$\Omega_{0}$

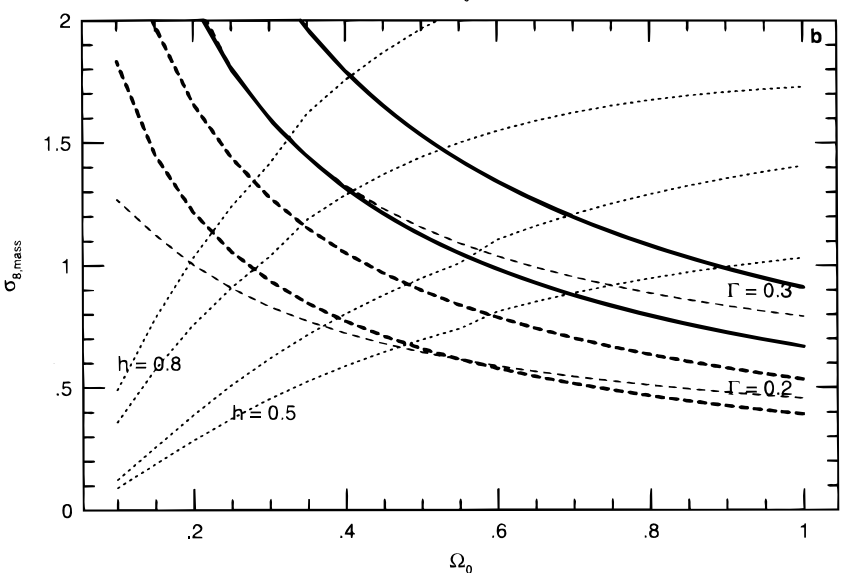

FIG. 12. - (a) Bias $b$ vs. $\Omega_{0}$ for $\Lambda$ CDM models where $\Omega_{0}+\Omega_{\Lambda}=1.2 \sigma$ error bands are shown for fixed shape parameters $\Gamma$ and fixed Hubble constants $h$. The lines $b=0.7$ and 1.3 roughly indicate the range of reasonable bias values. (b) $C O B E$-normalized $\sigma_{8 \text { mass }}$-values vs. $\Omega_{0}$ for the same models as in $(a)$. The unlabeled thin dashed lines indicate $95 \%$ confidence limits from cluster abundance constraints. See text for more details.

which are unbiased and which also meet the cluster constraints. An $\Omega_{0}=1, \Gamma=0.3$ model is unbiased but requires an unreasonably low $h \approx 0.3$ and is marginal with respect to cluster abundances.

OCDM.-The $\Gamma$ fit is the same as in the $\Lambda$ CDM case. We plot the corresponding $\Gamma=0.3$ and $h=0.5,0.8$ curves in the $b$ and $\sigma_{8 \text { mass }}$ versus $\Omega_{0}$ planes in Figure 13. The most dramatic difference in the open CDM case versus the flat $\Lambda \mathrm{CDM}$ case arises from the much weaker variation with $\Omega_{0}$ of the $C O B E$ normalization. The parameter space opens up a bit and for $0.4 \lesssim \Omega_{0} \lesssim 0.6$ there are models that satisfy $\Gamma=0.3$ and $0.5 \lesssim h \lesssim 0.8$, are unbiased, and match the cluster abundances.

CHDM.--In Figure 14 we plot our results, $b$ and $\sigma_{8 \text {, mass }}$ versus neutrino density $\Omega_{v}$, for two cases $h=0.5,0.8$. The $h=0.8$ results are strongly antibiased, $b \approx 0.3$, violate the cluster abundances, and therefore appear ruled out. On the other hand, the $h=0.5$ models are mildly antibiased, $b \approx 0.8$, and satisfy the cluster abundances for $\Omega_{v} \gtrsim 0.2$. The LCRS fit further constrains $\Omega_{v}$ to $0.2 \pm 0.1$ (the error is one sdom among the four subsamples) for the case $h=0.5$.

TCDM.-Here we plot $b$ and $\sigma_{8 \text { mass }}$ versus the spectral index $n$, again for the two cases $h=0.5$ and 0.8 , in Figure 15 . For $h=0.5$ and $n \approx 0.75$ the models are unbiased and also satisfy cluster abundances. The LCRS fit gives $n=0.7 \pm 0.2$. For $h=0.8$ we instead need $n \lesssim 0.6$ to meet
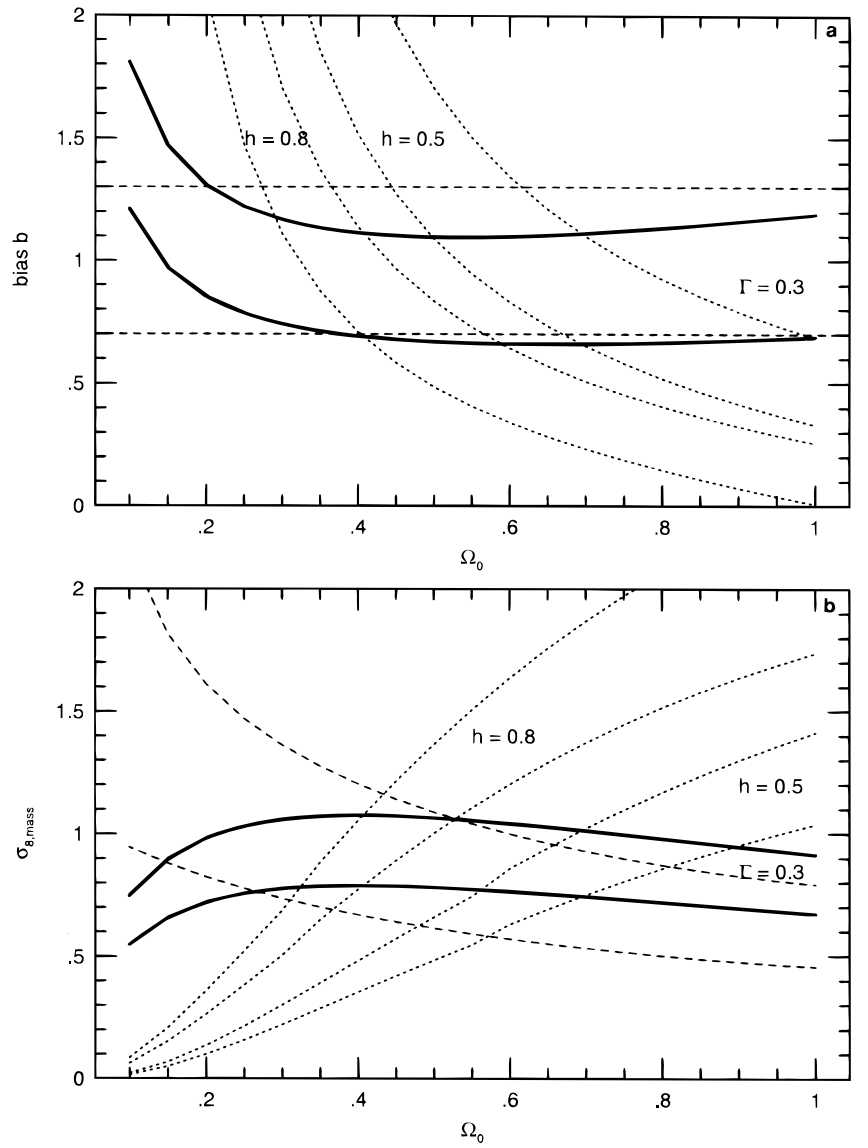

Fig. 13.-Similar to Fig. 12, except that open CDM models with $\Omega_{0}<1$ are being considered.

the constraints, but this appears to violate the direct $C O B E$ DMR fit to the spectral index, $n=1.2 \pm 0.3(1 \sigma$; Bennett et al. 1996).

In summary, all the simple CDM variants we have considered can provide models that simultaneously satisfy the LCRS large-scale power spectrum shape constraints, have Hubble constant $0.5 \lesssim h \lesssim 0.8$, give an approximately unbiased LCRS galaxy distribution on large scales, and meet the cluster abundance constraints. For each of our four CDM classes, we plot in Figure 16 specific example linear power spectra that meet the above criteria:

$$
\begin{array}{lrlll}
\Lambda \mathrm{CDM}: \Omega_{0}=0.5 & \Gamma=0.2 & h=0.5 & b=0.9 \\
\text { OCDM: } \Omega_{0}=0.5 & \Gamma=0.3 & h=0.65 & b=0.9 \\
\text { CHDM: } \Omega_{0}=1 & \Omega_{v}=0.2 & h=0.5 & b=0.8 \\
\text { TCDM: } \Omega_{0}=1 & n=0.7 & h=0.5 & b=1.3
\end{array}
$$

The model linear power spectra are shown both unconvolved and convolved, and compared to the corresponding LCRS results. Note the similarity in the shapes of the convolved power spectra that we are actually fitting. However, not surprisingly, the true unconvolved spectra do in fact differ more in shape, and they also show more power compared to the directly deconvolved LCRS power spectrum for $\lambda \gtrsim 200 h^{-1} \mathrm{Mpc}$. Also shown are corresponding COBE $1 \sigma$ error boxes, shifted to redshift space using equation (29) and the appropriate bias value.

The acceptable regions of our CDM model parameter space appear to be the following. Flat $\Omega_{0}=1, h=0.5$ 

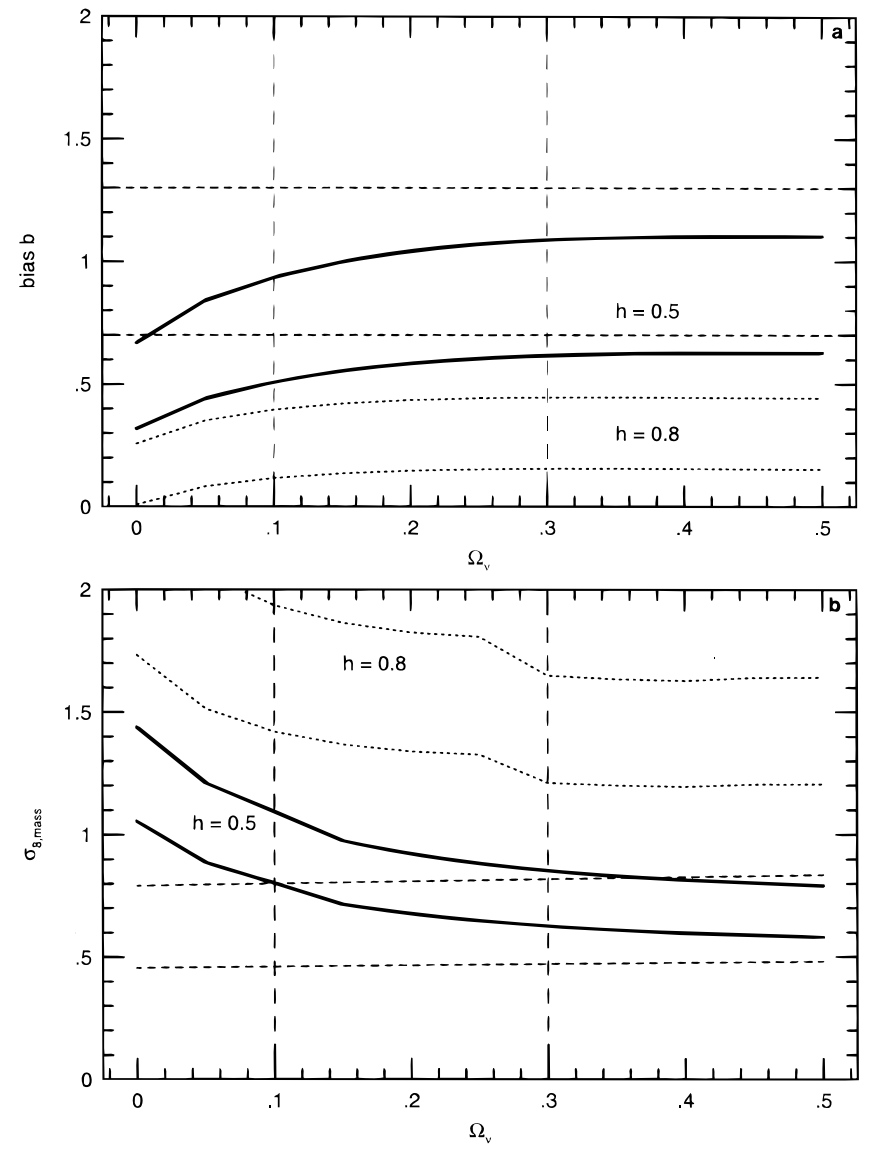

FIG. 14.- Similar to Fig. 12, except that $\Omega_{0}=1$ CHDM models with massive neutrinos of density $\Omega_{v}$ are being considered. The vertical lines indicate the LCRS best-fit range $\Omega_{v}=0.2 \pm 0.1(1 \sigma)$ for the case $h=0.5$.

models are still viable, if we add massive neutrinos with $\Omega_{v} \approx 0.2-0.3$ or a spectral tilt $n \approx 0.7-0.8$. Alternatively, flat $\Omega_{0}+\Omega_{\Lambda}=1$ CDM models with $\Gamma \approx 0.2$ and $\Omega_{0} \approx 0.4-0.5$ will also do, and similarly for open CDM models with $\Omega_{0} \approx 0.4-0.6$ and $\Gamma \approx 0.3$. We also note that these models can provide $\sigma_{8, \text { mass }}$-values near unity, which was suggested in the earlier comparison of our luminosity bias results with the predictions of Kauffmann et al. (1996). Similarly, these models can also meet the constraint $\sigma_{8 \text {, mass }} \Omega_{0}^{0.6} \simeq 0.7-0.8$ from peculiar velocity data (Kolatt \& Dekel 1996). Additionally, they also satisfy the $95 \%$ lower bound on the age of the universe, $12 \mathrm{Gyr}$, as determined from the ages of the oldest globular clusters (Chaboyer et al. 1996).

We have by no means attempted to exhaustively compare our results to all the possibilities (e.g., we can incorporate tilt in the open models, add gravity waves, and so on), but have simply explored some simple variations on the CDM theme, and have seen that there is already room for a number of such viable models. The next generation of very large redshift surveys, such as Sloan (Gunn \& Weinberg 1995) and 2DF (Ellis 1993) should provide us with improvements on the large-scale galaxy power spectrum (Vogeley 1995). In conjunction with improvements in degree-scale microwave anisotropy experiments (e.g., see reviews by Scott, Silk, \& White 1995; Bond 1996), the convergence of the large-scale structure and microwave background observational fronts will hopefully give us a more definitive answer. On the other hand, for smaller scales $\lambda<100 h^{-1}$ Mpc, $P(k)$ is already quite well constrained in the LCRS. In particular, any viable model must match these small-scale
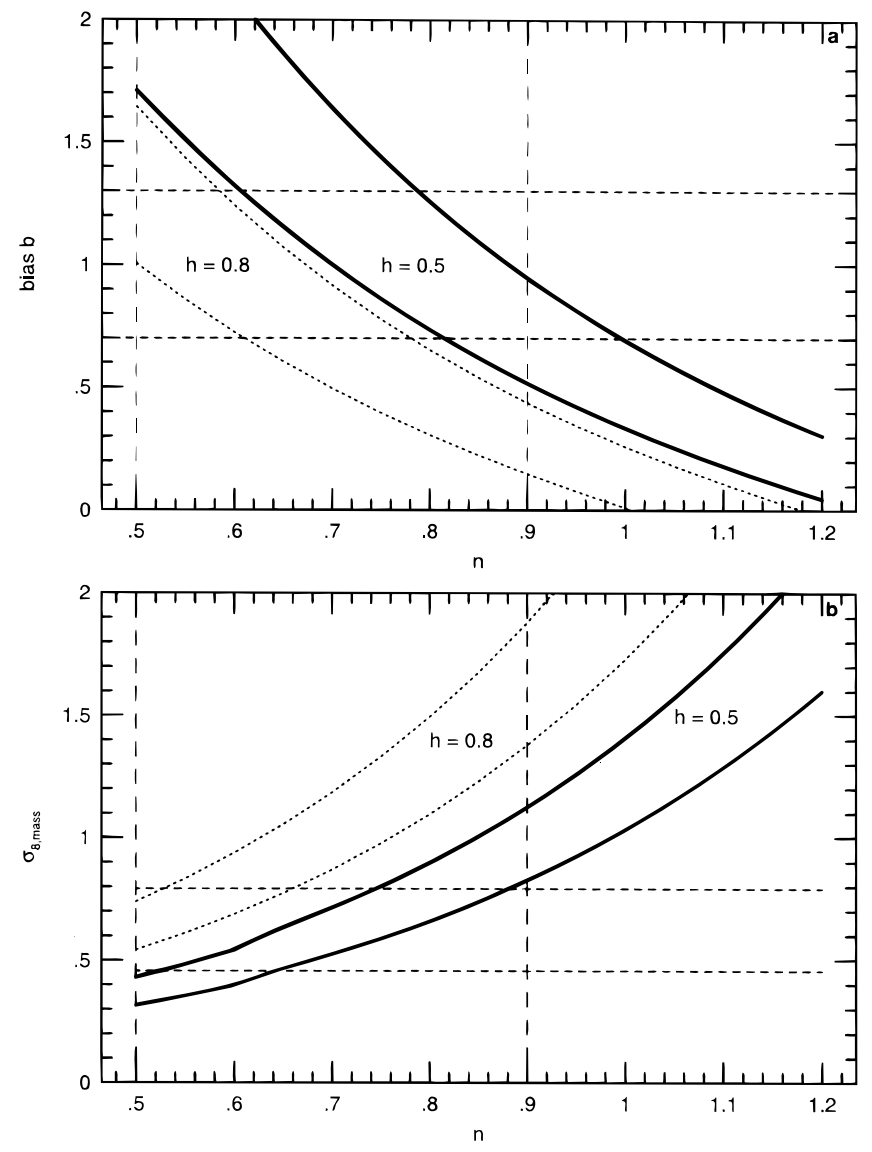

FIG. 15.-Similar to Fig. 12, except that $\Omega_{0}=1$ tilted CDM models with spectral index $n$ are being considered. The vertical lines indicate the LCRS best-fit range $n=0.7 \pm 0.2(1 \sigma)$ for the case $h=0.5$.

results, as we have seen earlier in the case of our two $N$-body models. The complication here is that galaxies exhibit type-dependent clustering differences (as is the case for our emission samples and for our super- $M^{*}$ galaxies), which will make it more difficult to interpret which types of galaxies are actually being simulated by the models, in as much as the process of galaxy formation is still not well understood, and there is freedom of choice in how one's "galaxies" are defined and represented in the simulations. The connection between the $N$-body models and the statistics of a galaxy sample will remain a weak link that will require a more thorough understanding of the types of galaxies selected by a particular survey and their relation to the underlying density fluctuations.

\section{CONCLUSIONS}

We have computed the power spectrum for a magnitudelimited sample of 19,305 Las Campanas survey galaxies, with average redshift $z=0.1$ and absolute magnitudes $-22.5<M<-18.5$, over scales $\lambda=5-400 h^{-1}$ Mpc. $P(k)$ may be approximated by a power law $P(k) \propto k^{n}$, with slopes $n=-1.8 \pm 0.1$ on small scales $\lambda=5-30 h^{-1} \mathrm{Mpc}$, and $n=1 \pm 1$ on the largest scales $\lambda \approx 200-400 h^{-1} \mathrm{Mpc}$. The change in slope of $P(k)$ is real and could be the result of a possible maximum in $P(k)$ observed at $\lambda \approx 100 h^{-1} \mathrm{Mpc}$, though our errors preclude a definitive detection of the turnover. The overall amplitude may be expressed as $\sigma_{8}=$ $1.0 \pm 0.1$ in redshift space. We have also compared our results to those from other redshift surveys. For $\lambda \lesssim 100$ $h^{-1} \mathrm{Mpc}$, the LCRS $P(k)$ is similar in shape to that of the 

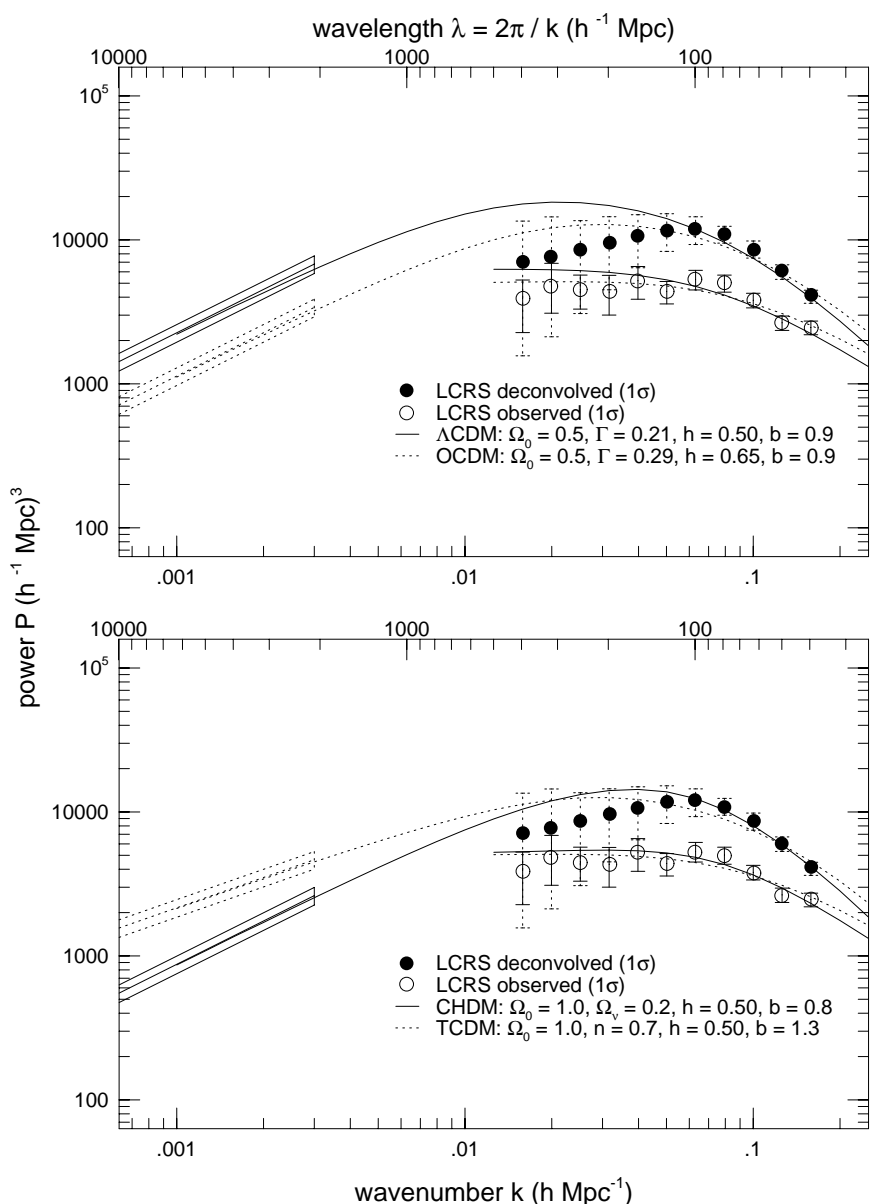

FIG. 16.-LCRS power spectrum and the fits to it using the linear CDM models described in the text, shown both in convolved and unconvolved form. Also shown are corresponding COBE $1 \sigma$ error boxes, transformed into redshift space using the indicated bias values. See text for details.

other samples, but with some differences in amplitude that may reflect intrinsic clustering differences for galaxies of different luminosities or types (below). For $\lambda \gtrsim 100 h^{-1}$ Mpc, the LCRS results are consistent with those of other surveys given the large errors among all the surveys on these scales. The overall LCRS results agree best with that from the combined SSRS2 + CfA2 redshift surveys.

We find evidence in the LCRS for the dependence of clustering strength on galaxy type. A sample of LCRS emission galaxies with [O II] $\lambda 3727$ equivalent widths $\geq 5 \AA$ are about $30 \%$ less clustered than the full LCRS sample on scales $\lambda \lesssim 100 h^{-1} \mathrm{Mpc}$. This may reflect an intrinsically weaker clustering strength for spiral galaxies, in qualitative agreement with the weaker clustering of $I R A S$ versus optically selected galaxies. We also compute the power spectra of volume-limited LCRS samples to test for evidence of luminosity bias. We find that LCRS galaxies in the range $-18.5>M-5 \log h>-21$ appear to be clustered similarly, over scales $\lambda \approx 5-150 h^{-1} \mathrm{Mpc}$. However, more luminous galaxies, which lie about 1 magnitude brighter than $M_{\text {LCRS }}^{*}=-20.3+5 \log h$, exhibit about a $50 \%$ stronger clustering amplitude, in agreement with similar results seen in the CfA2 and SSRS2 surveys and the qualitative expectations of biased galaxy formation scenarios.

Our full nonlinear power spectrum is intermediate between that of two CDM $N$-body models, an open $\Omega_{0} h=$
0.2 model and a standard $\Omega_{0} h=0.5$ model, both normalized to $\sigma_{8}=1$ for galaxies. The models agree well with the LCRS results on small scales $\lambda \lesssim 50 h^{-1} \mathrm{Mpc}$, but neither model provides a completely satisfactory fit to the full LCRS power spectrum. To explore more of model parameter space, we then fit the large-scale LCRS power, on approximately linear scales $\lambda \gtrsim 40 h^{-1} \mathrm{Mpc}$, to several classes of linear CDM models. We find that a number of viable models remain that can simultaneously satisfy constraints provided by the LCRS power spectrum, the Hubble constant range $0.5 \lesssim h \lesssim 0.8$, cluster abundance results, and the reasonable assumption that LCRS galaxies are approximately unbiased on large scales, relative to the mass normalization provided by the 4 year $C O B E$ DMR data. These models include (1) flat $\Omega_{\Lambda}+\Omega_{0}=1$ CDM models with $\Omega_{0} \approx 0.4-0.5$ and shape parameter $\Gamma \approx \Omega_{0} h \approx 0.2 ;(2)$ open CDM models with $\Omega_{0} \approx 0.4-0.6$ and $\Gamma \approx 0.3$; (3) flat $\Omega_{0}=$ 1 models with CDM plus massive neutrinos of density $\Omega_{v} \approx 0.2-0.3$; and (4) flat $\Omega_{0}=1$ CDM models with a spectral tilt $n \approx 0.7-0.8$

The present situation for power spectrum measurements from redshift surveys appears to fall into two regimes. On scales $\lambda \lesssim 100 h^{-1} \mathrm{Mpc}$, the power spectrum is well determined, and various surveys have consistently detected differences in the clustering strengths of different galaxy populations, though the latter effect may complicate interpretation of model predictions. On the largest scales $\lambda \gtrsim$ $100 h^{-1} \mathrm{Mpc}$, however, the errors in $P(k)$ are still large. We have not been able to conclusively rule out any of the popular classes of CDM models, though we have begun to constrain their parameter space. Nonetheless, the observational picture should improve when the next generation of very large redshift surveys come on line, and as the degreescale microwave background observations progress. In the meanwhile, the Las Campanas results have already provided constraints on $P(k)$ on both large and small scales, complementary to, and largely independent of, earlier results for smaller and shallower samples. Furthermore, the typedependent clustering differences observed in the survey should provide useful observational constraints on models that seek to reproduce accurately the clustering properties and formation histories for a realistic mix of galaxy types. Toward this end, interested readers are invited to obtain the Las Campanas Redshift Survey catalog, now publicly available at http://manaslu.astro.utoronto.ca/lin/lcrs.html.

Note added in manuscript.-Power spectrum results for the Stromlo-APM redshift survey (Tadros \& Efstathiou 1996) appeared after submission of the present paper. Time does not permit a detailed comparison, but we note that the Stromlo-APM authors report that their results are consistent with those of the CfA2 101 sample (Park et al. 1994), and that they also find evidence for the stronger clustering of galaxies brighter than $M^{*}$.

We thank Changbom Park for providing the $N$-body models, Michael Vogeley for useful discussions and for providing the SSRS2 + CfA2 results, Hume Feldman for the QDOT results, and Tsafrir Kolatt for helpful comments on the manuscript. The Las Campanas Redshift Survey has been supported by NSF grants AST 87-17207, AST 8921326, and AST 92-20460. H. L. also acknowledges support from NASA grant NGT-51093 and support at the University of Toronto where this paper was completed. 


\section{REFERENCES}

Bardeen, J. M., Bond, J. R., Kaiser, N., \& Szalay, A. S. 1986, ApJ, 304, 15

Bardeen, J. M., Steinhardt, P. J., \& Turner, M. S. 1983, Phys. Rev. D, 28 679

Baugh, C. M., \& Efstathiou, G. 1993, MNRAS, 265, 145 1994, MNRAS, 267, 323

Benoist, C., Maurogordato, S., da Costa, L. N., Cappi, A., \& Schaeffer, R. 1996, ApJ, 472, in press

Bennett, C. L., et al. 1996, ApJ, 464, L1

Blumenthal, G., Faber, S. M., Primack, J. R., \& Rees, M. J. 1984, Nature 311,517

Bond, J. R. 1996, in The Evolution of the Universe, Dahlem Workshop Report ES 19, in press

Bond, J. R., \& Efstathiou, G. 1984, ApJ, 285, L45

Broadhurst, T. J., Ellis, R. S., Koo, D. C., \& Szalay, A. S. 1990, Nature, 343, 726

Chaboyer, B., Demarque, P., Kernan, P. J., \& Krauss, L. M. 1996, Science, 271, 957

Copi, C. J., Schramm, D. N., \& Turner, M. S. 1995, Science, 267, 192

da Costa, L. N., et al. 1994a, ApJ, 424, L1

da Costa, L. N., Vogeley, M. S., Geller, M. J., Huchra, J. P., \& Park, C. 1994b, ApJ, 437, L1

Davis, M., Efstathiou, G., Frenk, C. S., \& White, S. D. M. 1985, ApJ, 292 371

Davis, M., \& Geller, M. J. 1976, ApJ, 208, 13

Doroshkevich, A. G., Tucker, D. L., Oemler, A., Kirshner, R. P., Lin, H. Shectman, S. A., Landy, S. D., \& Fong, R. 1996, MNRAS, in press

Efstathiou, G., Bond, J. R., \& White, S. D. M. 1992, MNRAS, 258, 1 P

Ellis, R. S. 1993, in ASP Conf. Ser. 43, Sky Surveys: Protostars to Protogalaxies, ed. B. T. Soifer (San Francisco: ASP), 165

Feldman, H. A., Kaiser, N., \& Peacock, J. A. 1994, ApJ, 426, 23

Fisher, K. B., Davis, M., Strauss, M. A., Yahil, A., \& Huchra, J. P. 1993 ApJ, 402, 42

Freedman, W. L., et al. 1994, Nature, 371, 757

Gunn, J. E., \& Weinberg, D. H. 1995, in Proc. 35th Herstmonceux Conf Wide-Field Spectroscopy and the Distant Universe, ed. S. J. Maddox \& A. Aragón-Salamanca (Singapore: World Scientific), 3

Hamilton, A. J. S. 1988, ApJ, 331, L59

Hockney, R. W., \& Eastwood, J. W. 1981, Computer Simulation Using Particles (New York: McGraw-Hill)

Jacoby, G. H., et al. 1992, PASP, 104, 599

Jain, B., Mo, H. J., \& White, S. D. M. 1995, MNRAS, 276, L25

Kaiser, N. 1987, MNRAS, 227, 1

Kauffmann, G., Nusser, A., \& Steinmetz, M. 1996, MNRAS, submitted

Kennicutt, R. C. 1992, ApJ, 388, 310

Kolatt, T., \& Dekel, A. 1996, ApJ, submitted

Landy, S. D., Shectman, S. A., Lin, H., Kirshner, R. P., Oemler, A., \& Tucker, D. L. 1996, ApJ, 456, L1

Liddle, A. R., Lyth, D. H., Roberts, D., \& Viana, P. T. P. 1996a, MNRAS 278,644

Liddle, A. R., Lyth, D. H., Schaefer, R. K., Shafi, Q., \& Viana, P. T. P. 1996b, MNRAS, 281, 531

Liddle, A. R., Lyth, D. H., Viana, P. T. P., \& White, M. 1996c, MNRAS, in press

Lightman, A. P., \& Schechter, P. L. 1990, ApJS, 74, 831

Lin, H. 1995, Ph.D. thesis, Harvard Univ.

Lin, H., Kirshner, R. P., Shectman, S. A., Landy, S. D., Oemler, A., Tucker, D. L., \& Schechter, P. L. 1996, ApJ, 464, 60

Loveday, J., Efstathiou, G., Maddox, S. J., \& Peterson, B. A. 1996, ApJ, 468,1
Loveday, J., Maddox, S. J., Efstathiou, G., \& Peterson, B. A. 1995, ApJ, 442,457

Lucy, L. B. 1974, AJ, 79, 745

Lyth, D. H., \& Liddle, A. R. 1996, in Proc. Journee Cosmologie, in press

Mann, R. G., Saunders, W., \& Taylor, A. 1996, MNRAS, 279, 636

Marzke, R. O., Huchra, J. P., \& Geller, M. J. 1994, ApJ, 428, 43

Mo, H. J., Einasto, M., Xia, X. Y., \& Deng, Z. G. 1992, MNRAS, 255, 382

Oemler, A., Tucker, D. L., Kirshner, R. P., Lin, H., Shectman, S. A., \& Schechter, P. L. 1993, in ASP Conf. Ser. 51, Observational Cosmology, ed. G. Chincarini, A. Iovino, T. Maccacaro, \& D. Maccagni (San Francisco: ASP), 81

Park, C. 1991, MNRAS, 251, 167

Park, C., Gott, J. R., \& da Costa, L. N. 1992, ApJ, 392, L51

Park, C., Vogeley, M. S., Geller, M. J., \& Huchra, J. P. 1994, ApJ, 431, 569

Peacock, J. A. 1992, in New Insights into the Universe, Proc. Valencia Summer School, ed. V. J. Martinez, M. Portilla, \& D. Saez (Berlin: Springer), 1

Peacock, J. A., \& Dodds, S. J. 1994, MNRAS, 267, 1020

Peacock, J. A., \& Nicholson, D. 1991, MNRAS, 253, 307

Peebles, P. J. E. 1980, The Large-Scale Structure of the Universe (Princeton: Princeton Univ. Press)

Pogosyan, D. Yu., \& Starobinsky, A. A. 1995, ApJ, 447, 465

Riess, A. G., Press, W. H., \& Kirshner, R. P. 1995, ApJ, 438, L17

Schechter, P. 1976, ApJ, 203, 297

Schmidt, B. P., Kirshner, R. P., Eastman, R. G., Phillips, M. M., Suntzeff,

N. B., Hamuy, M., Maza, J., \& Avilés, R. 1994, ApJ, 432, 42

Scott, D., Silk, J., \& White, M. 1995, Science, 268, 829

Shectman, S. A., Landy, S. D., Oemler, A., Tucker, D. L., Kirshner, R. P.

Lin, H., \& Schechter, P. L. 1995, in Proc. 35th Herstmonceux Conf., Wide-Field Spectroscopy and the Distant Universe, ed. S. J. Maddox \&

A. Aragón-Salamanca (Singapore: World Scientific), 98 .1996, ApJ, 470, in press

Shectman, S. A., Schechter, P. L., Oemler, A., Tucker, D., Kirshner, R. P \& Lin, H. 1992, in Clusters and Superclusters of Galaxies, ed. A. C. Fabian (Dordrecht: Kluwer), 351

Smoot, G. F., et al. 1992, ApJ, 396, L1

Strauss, M. A., Davis, M., Yahil, Y., \& Huchra, J. P. 1992, ApJ, 385, 421

Tadros, H., \& Efstathiou, G. 1995, MNRAS, 276, L45

1996, MNRAS, submitted

Tegmark, M. 1995, ApJ, 455, 429

Tucker, D. L. 1994, Ph.D. thesis, Yale Univ.

Tucker, D. L., Oemler, A., Kirshner, R. P., Lin, H., Shectman, S. A., Landy, S. D., Schechter, P. L., Müller, V., Gottlöber, S., \& Einasto, J. 1996 , MNRAS, submitted

Viana, P. T. P., \& Liddle, A. R. 1996, MNRAS, 281, 323

Vogeley, M. S. 1995, in Proc. 35th Herstmonceux Conf., Wide-Field Spectroscopy and the Distant Universe, ed. S. J. Maddox \& A. AragónSalamanca (Singapore: World Scientific), 142

Vogeley, M. S., Park, C., Geller, M. J., \& Huchra, J. P. 1992, ApJ, 391, L5

Vogeley, M. S., \& Szalay, A. S. 1996, ApJ, 465, 34

Weinberg, D. H. 1995, in Proc. 35th Herstmonceux Conf., Wide-Field Spectroscopy and the Distant Universe, ed. S. J. Maddox \& A. AragónSalamanca (Singapore: World Scientific), 129

White, S. D. M., Davis, M., Efstathiou, G., \& Frenk, C. S. 1987, Nature, 330,451

Zurek, W. H., Quinn, P. J., Salmon, J. K., \& Warren, M. S. 1994, ApJ, 431, 